\title{
Approximation of a Class of Incompressible Third Grade Fluids Equations
}

\author{
Zeqi Zhu, ${ }^{1}$ Hongxia Li, ${ }^{2}$ Jianjun $\mathrm{Fu}^{3}{ }^{3}$ and Qian Sheng ${ }^{1}$ \\ ${ }^{1}$ State Key Laboratory of Geomechanics and Geotechnical Engineering, Institute of Rock and Soil Mechanics, \\ Chinese Academy of Sciences, Wuhan, Hubei 430071, China \\ ${ }^{2}$ College of Science, Naval University of Engineering, Wuhan, Hubei 430033, China \\ ${ }^{3}$ Power China Zhongnan Engineering Corporation Limited, Changsha, Hunan 410014, China
}

Correspondence should be addressed to Zeqi Zhu; zhuzeqi2014@163.com

Received 21 October 2014; Revised 21 December 2014; Accepted 22 December 2014

Academic Editor: Ivan Area

Copyright (C) 2015 Zeqi Zhu et al. This is an open access article distributed under the Creative Commons Attribution License, which permits unrestricted use, distribution, and reproduction in any medium, provided the original work is properly cited.

This paper discusses the approximation of weak solutions for a class of incompressible third grade fluids equations. We first introduce a family of perturbed slightly compressible third grade fluids equations (depending on a positive parameter $\epsilon$ ) which approximate the incompressible equations as $\epsilon \rightarrow 0^{+}$. Then we prove the existence and uniqueness of weak solutions for the slightly compressible equations and establish that the solutions of the slightly compressible equations converge to the solutions of the incompressible equations.

\section{Introduction}

Fluids of differential type form an important class of nonNewtonian fluids. The fluids of grade $n$, introduced by Rivlin and Ericksen [1], are the fluids for which the stress tensor is a polynomial of degree $n$ in the first $n$ Rivlin-Ericksen tensor defined recursively by

$$
\begin{gathered}
A_{1}(u)=A(u)=\nabla u+(\nabla u)^{T}, \\
A_{k+1}(u)=\frac{D}{D t} A_{k}(u)+(\nabla u)^{T} A_{k}(u)+A_{k}(u) \nabla u, \\
k=1,2, \ldots,
\end{gathered}
$$

where $D / D t=\partial / \partial t+u \times \nabla$ denotes the material derivative and $(\nabla u)^{T}$ the transposition of the Jacobian matrix $\nabla u$. In [1], the constitutive relation of a particular of fluids of grade $n$ is given by

$$
T=-p I+F\left(A_{1}, A_{2}, \ldots, A_{n}\right),
$$

where $I$ is the identity matrix of degree $n$ and $F$ is an isotropic polynomial of degree $n$.

There are some references on the existence, uniqueness, and asymptotic behavior of solutions for second and third grade fluids equations; see, for example, [2-10] and the references therein. In [2-4], Azia et al. use the symmetry approach to obtain the analytical solutions of the third grade fluid equations. In [5], Busuioc and Iftime studied the existence of solutions to the following third grade fluids equations:

$$
\begin{gathered}
\partial_{t} v+u \times \nabla v+\sum_{j} v_{j} \nabla u_{j}-v \Delta u \\
=\left(\alpha_{1}+\alpha_{2}\right) \operatorname{div}\left(A^{2}(u)\right)+\beta \operatorname{div}\left(|A(u)|^{2} A(u)\right)-\nabla p, \\
v=u-\alpha_{1} \Delta u, \\
\operatorname{div} u=0, \\
u(x, 0)=u_{0}(x),
\end{gathered}
$$

in $\mathbb{R}^{n}(n=2,3)$, with the coefficients $\nu, \alpha_{1}, \alpha_{2}$, and $\beta$ satisfying the following hypotheses:

$$
\nu \geq 0, \quad \alpha_{1}>0, \quad \beta \geq 0, \quad\left|\alpha_{1}+\alpha_{2}\right| \leq \sqrt{24 \nu \beta} .
$$

Also, Busuioc and Iftimie in [5] proved that the third grade fluids equations (3) possess a global solution if the initial value 
is in $H^{2}\left(\mathbb{R}^{n}\right)(n=2,3)$ and established the uniqueness of solutions for (3) when $n=2$. Recently, Hamza and Paicu studied in [7] a particular case of the third grade fluids equations (3) in $\mathbb{R}^{3}$, where they assume $\alpha_{1}=0$. Then (3) become

$$
\begin{gathered}
\partial_{t} u+u \times \nabla u-v \Delta u-\alpha_{2} \operatorname{div}\left(A^{2}(u)\right) \\
-\beta \operatorname{div}\left(|A(u)|^{2} A(u)\right)+\nabla p=0, \\
\operatorname{div} u=0, \\
u(x, 0)=u_{0}(x),
\end{gathered}
$$

with the following assumptions on the coefficients $v, \alpha_{1}, \alpha_{2}$, and $\beta$ :

$$
\alpha_{1}=0, \quad \beta>0, \quad \alpha=\alpha_{2} \quad \text { with }|\alpha|<\sqrt{2 \nu \beta},
$$

Hamza and Paicu in [7] proved the existence and uniqueness, as well as the stability (when $|\alpha|<\sqrt{\nu \beta / 2}$ ), of global solutions for (5) with natural regularity assumption on the initial data belonging to the energy space $\mathbb{L}^{2}\left(\mathbb{R}^{3}\right)$. They also proved that if the initial datum belongs to $\mathbb{H}^{1}\left(\mathbb{R}^{3}\right)$, then the solution belongs to $\mathbb{T}^{1}\left(\mathbb{R}^{3}\right)$ for any positive time and they gave a control of the $\mathbb{M}^{1}$ norm of the solution. Very recently, Paicu et al. proved in [8] the regularity of the global attractor and finitedimensional behavior for the second grade fluids equations in the two-dimensional torus.

In the present paper, we first discuss the approximation of the incompressible third grade fluids equations (5) in threedimensional bounded smooth domain, via the following slightly compressible third grade fluids equations depending on a parameter $\epsilon \in(0,1]$ :

$$
\begin{aligned}
\partial_{t} u_{\epsilon}+u_{\epsilon} \times \nabla u_{\epsilon}+\frac{1}{2}\left(\operatorname{div} u_{\epsilon}\right) u_{\epsilon}-v \Delta u_{\epsilon} \\
=\alpha \operatorname{div}\left(A^{2}\left(u_{\epsilon}\right)\right)+\beta \operatorname{div}\left(\left|A\left(u_{\epsilon}\right)\right|^{2} A\left(u_{\epsilon}\right)\right)-\nabla p_{\epsilon} \\
\epsilon \partial_{t} p_{\epsilon}+\operatorname{div} u_{\epsilon}=0, \quad x \in \Omega \subset \mathbb{R}^{3}, \quad t>0
\end{aligned}
$$

with initial-boundary value conditions:

$$
\left.u_{\epsilon}\right|_{\partial \Omega}=0, \quad u_{\epsilon}(x, 0)=u_{0}(x), \quad p_{\epsilon}(x, 0)=p_{0} .
$$

Compared with the incompressible third grade fluids equations (5), (7)-(8) overcome the computational difficulties connected with the constraint " $\operatorname{div} u=0$." It is easier to approximate than the original incompressible equations as the constraint " $\operatorname{div} u=0$ " has been replaced by the evolution equation (8). The third grade fluids equations (7)-(8) are called "slightly compressible" for the parameter $\epsilon$ will be taken to tend to zero. Obviously, the perturbed compressible third grade fluids equations (7)-(9) turn to be the incompressible third grade fluids equations (5) as $\epsilon=0$. Following this clue, the questions are now the following.

(i) Does the solution of the initial-boundary value problem of perturbed compressible third grade fluids equations (7)-(9) uniquely exist? (ii) Does the solution $\left(u_{\epsilon}, p_{\epsilon}\right)$ of (7)-(9) converge to the solution $(u, p)$ of the incompressible third grade fluids equations (5) as $\epsilon \rightarrow 0^{+}$?

The purpose of this paper is to give answers to the above two questions. When using the classical Faedo-Galerkin method to prove the existence of a weak solution for the compressible third grade fluid equations (7)-(9), the main difficulty (compared with the incompressible case) comes from the presence of the term $\nabla p_{\epsilon}$ in the slightly compressible third grade fluids equations. Due to the presence of the term $\nabla p_{\epsilon}$, the argument for the incompressible case (see, e.g., [7]) to obtain the bound of the derivative sequence $\left\{\partial u_{\epsilon n} / \partial t\right\}_{n=1}^{\infty}$ seems not applicable. This is caused essentially by the compressibility of the fluids. We know in the incompressible case $\operatorname{div} u=0$ and we can take $H$ or $V$ (see the notation in Section 2) as the phase space. Naturally, the term $\nabla p$ will disappear under the projection of the Helmhloz-Leray projector from $\mathbb{L}^{2}(\Omega)$ to $H$. While the fluids are compressible, $\operatorname{div} u \neq 0$ and we shall take $\mathbb{L}^{2}(\Omega)$ or $\mathbb{T}_{0}^{1}(\Omega)$ (or other Sobolev spaces) as the phase space. So the term $\nabla p_{\epsilon}$ will not disappear. To overcome this difficulty, we will use the Fourier transform (in time $t$ ) technique to obtain the boundedness of the fractional derivative in time variable $t$ of the sequence $\left\{u_{\epsilon n}\right\}_{n=1}^{\infty}$.

The paper is organized as follows. In Section 2, we give some notations first and then the existence and uniqueness of weak solutions for (5). In Section 3, we describe the weak formulation of (7)-(9) and then prove the existence and uniqueness of its weak solution. In Section 4, we show how the solutions of the slightly compressible third grade fluids equations converge to the solutions of the corresponding incompressible third grade fluids equations.

\section{Preliminaries}

In this paper, we denote by $c$ the generic constant that can take different values in different places. $\mathbb{L}^{p}(\Omega)=L^{p}(\Omega) \times$ $L^{p}(\Omega) \times L^{p}(\Omega)$ is the $3 \mathrm{D}$ Lebesgue space with norm $\|\cdot\|_{\Omega^{p}(\Omega)}$ and $\mathbb{H}_{0}^{m}(\Omega)=H_{0}^{m}(\Omega) \times H_{0}^{m}(\Omega) \times H_{0}^{m}(\Omega)$ is the $3 \mathrm{D}$ Sobolev space with norm $\|\cdot\|_{\mathbb{Q}_{0}^{m}(\Omega)}$ and dual space $\mathbb{M}^{-m}(\Omega)$, where $L^{p}(\Omega)=L^{p}$ and $H^{m}(\Omega)=H^{m}$ are the usual $L^{p}$-Lebesgue space and Sobolev space on $\Omega$, respectively. When $p=2$, we denote $\|\cdot\|_{\mathbb{L}^{2}(\Omega)}=\|\cdot\|$ and also $\|\cdot\|_{L^{2}(\Omega)}=\|\cdot\|$ if there is no confusion. $\mathbb{W}_{0}^{1,4}(\Omega)$ is the usual $3 \mathrm{D}$ Sobolev space and $\mathbb{W}^{-1,4 / 3}(\Omega)$ is its dual space (see [11]). Write

$$
\begin{gathered}
\mathscr{V}=\left\{\phi: \text { the } 3 \mathrm{D} \text { vector-valued } \mathscr{C}_{0}^{\infty}(\Omega)\right. \\
\text { functions and } \nabla \cdot \phi=0\},
\end{gathered}
$$

and denote by $H$ and $V$ the closure space of $\mathscr{V}$ in $\mathbb{L}^{2}$ norm and in $\mathbb{H}_{0}^{1}$ norm, respectively. $(\cdot, \cdot)$ is the inner product of $H$ (or $\mathbb{L}^{2}(\Omega), L^{2}(\Omega)$ ), and $\langle\cdot, \cdot\rangle$ is the dual pairing between $V$ and $V^{\prime}$ (the dual space of $V$ ), or between $\mathbb{H}_{0}^{m}(\Omega)$ and $\mathbb{\boxplus}^{-m}(\Omega)$, or between $\mathbb{W}_{0}^{1,4}(\Omega)$ and $\mathbb{W}^{-1,4 / 3}(\Omega)$. Note

$$
\mathbb{W}_{0}^{1,4}(\Omega) \hookrightarrow \mathbb{H}_{0}^{1}(\Omega) \subset V \hookrightarrow H \subset \mathbb{L}^{2}(\Omega)
$$


To put (5) and (7)-(9) into abstract forms, respectively, we now introduce some operators. Firstly, set $\mathscr{L}=-\Delta$ (which is taken with zero boundary conditions). Then, using integration by parts, we have

$$
\langle\mathscr{L} u, v\rangle=(\nabla u, \nabla v), \quad \forall u, v \in \mathbb{H}_{0}^{1}(\Omega) \text { or } V .
$$

Secondly, we define a continuous trilinear form (see, e.g., [12]) $b(\cdot, \cdot, \cdot)$ on $\mathbb{H}_{0}^{1}(\Omega)$ (and in particular on $V$ ) by

$$
b(u, v, w)=\sum_{j, k=1}^{3} \int_{\Omega} u_{j} \frac{\partial v_{k}}{\partial x_{j}} w_{k} \mathrm{~d} x, \quad u, v, w \in \mathbb{U}_{0}^{1}(\Omega) \text { or } V .
$$

If $u \in V$, one can check

$$
\begin{array}{r}
b(u, v, w)=-b(u, w, v), \quad b(u, v, v)=0, \\
\forall v, w \in \mathbb{Q}_{0}^{1}(\Omega) .
\end{array}
$$

For $u, v \in \mathbb{H}_{0}^{1}(\Omega)$ (resp., $V$ ), we denote by $B(u, v)$ the element of $\mathbb{H}^{-1}(\Omega)\left(\right.$ resp., $\left.V^{\prime}\right)$ defined by $\langle B(u, v), w\rangle=b(u, v, w)$, for any $w \in \mathbb{Q}_{0}^{1}(\Omega)$ (resp., $V$ ), and set

$$
B(u)=B(u, u), \quad \forall u \in \mathbb{H}_{0}^{1}(\Omega)(\text { resp., } V) .
$$

The scalar product of two matrices $A=\left(a_{j k}\right)_{n \times n}$ and $B=$ $\left(b_{j k}\right)_{n \times n}$ is denoted by $A \times B=\sum_{j, k=1}^{n} a_{j k} b_{j k}$. We set $|A|^{2}=A \times$ $A=\sum_{j, k=1}^{n} a_{j k}^{2}$ and $(\operatorname{div} A)_{j}=\sum_{k=1}^{n} \partial_{k} a_{j k}$ for $j=1,2, \ldots, n$. We now define

$$
J(u)=-\alpha \operatorname{div}\left(A^{2}(u)\right)
$$

then the operator $J(u)$ maps $\mathbb{W}_{0}^{1,4}(\Omega)$ to $\mathbb{H}^{-1}(\Omega)$ via

$$
\langle J(u), v\rangle=\alpha \int_{\Omega} A^{2}(u) \times \nabla v \mathrm{~d} x, \quad \forall u \in \mathbb{W}_{0}^{1,4}(\Omega) .
$$

Also define

$$
K(u)=-\operatorname{div}\left(|A(u)|^{2} A(u)\right) ;
$$

then $K(u)$ maps $\mathbb{W}_{0}^{1,4}(\Omega)$ to $\mathbb{W}^{-1,4 / 3}(\Omega)$ by

$$
\langle K(u), v\rangle=\int_{\Omega}|A(u)|^{2} A(u) \times \nabla v \mathrm{~d} x, \quad \forall u \in \mathbb{W}_{0}^{1,4}(\Omega) .
$$

Using the notations and operators introduced above, we can express the weak formulation of (5) in the solenoidal field as

$$
\begin{gathered}
\partial_{t} u+v \mathscr{L} u+B(u)+J(u)+\beta K(u)=0, \\
x \in \Omega, \quad t>0, \\
\operatorname{div} u=0, \\
u(x, 0)=u_{0}(x) .
\end{gathered}
$$

Problem 1. Let $T>0$, for any given $u_{0} \in H$, to find $u$ satisfying

$$
\begin{gathered}
u \in L^{\infty}(0, T ; H) \cap L^{2}(0, T ; V) \cap L^{4}\left(0, T ; \mathbb{W}_{0}^{1,4}(\Omega)\right), \\
\partial_{t} u+v \mathscr{L} u+B(u)+J(u)+\beta K(u)=0, \\
\operatorname{in} \mathscr{D}^{\prime}\left(0, T ; \mathbb{W}^{-1,4 / 3}(\Omega)\right), \\
u(x, 0)=u_{0} .
\end{gathered}
$$

Lemma 2. Let the coefficients $\nu, \alpha$, and $\beta$ satisfy condition (6). Then Problem 1 possesses a unique solution.

The proof of this lemma is similar with that of [7], and we omit it here.

Remark 3. Let $\nu, \alpha$, and $\beta$ satisfy condition (6). Then for any given $u_{0} \in H$, for each solution $u$ obtained by Lemma 2 , there exists a unique pressure $p$ corresponding to $u$ and, for each $t \epsilon$ $[0, T], p(t) \in L^{2}(\Omega)$. Moreover, $\nabla p \in \mathscr{C}\left([0, T] ; \mathbb{H}^{-1}(\Omega)\right)$ and hence (one can refer to [13, Page 307] for a similar derivation)

$$
p \in \mathscr{C}\left([0, T] ; L^{2}(\Omega)\right),
$$

which satisfies in the sense of distribution that

$$
\begin{array}{r}
u^{\prime}+v \mathscr{L} u+B(u)+J(u)+\beta K(u)+\nabla p=0 . \\
\left(u^{\prime}=\partial_{t} u\right) .
\end{array}
$$

Therefore, for any $w \in \mathbb{W}_{0}^{1,4}(\Omega)$, there holds

$$
\left\langle u^{\prime}+v \mathscr{L} u+B(u)+J(u)+\beta K(u)+\nabla p, w\right\rangle=0,
$$

and for any $\varphi(t) \in \mathscr{C}_{0}^{\infty}(0, T)$,

$$
\begin{gathered}
-\int_{0}^{T}\langle u(t), w\rangle \varphi^{\prime}(t) \mathrm{d} t+v \int_{0}^{T}(\nabla u(t), \nabla w) \varphi(t) \mathrm{d} t \\
+\int_{0}^{T}\langle B(u(t)), w \varphi(t)\rangle \mathrm{d} t \\
=-\int_{0}^{T}\langle J(u(t))+\beta K(u(t)), w \varphi(t)\rangle \mathrm{d} t, \\
\forall w \in \mathbb{W}_{0}^{1,4}(\Omega) .
\end{gathered}
$$

Remark 4. If $u$ merely belongs to $L^{2}(0, T ; V)$, condition (23) needs not make sense. But if $u \in L^{2}(0, T ; V)$ and satisfies (26)(27), then we will show that

$$
u^{\prime} \in L^{4 / 3}\left(0, T ; \mathbb{W}^{-1,4 / 3}(\Omega)\right) .
$$

By the classical embedding theorem (see $[13,14])$, we infer from (21) and (28) that $u \in \mathscr{C}([0, T] ; H)$. Therefore, (23) is meaningful. 


\section{The Existence and Uniqueness of Solutions for Slightly Compressible Third Grade Fluids Equations}

In this section, we first give a description of the slightly compressible third grade fluids equations and then prove the existence and uniqueness of its weak solutions.

Since our ultimate purpose is to investigate the convergence of solutions of the slightly compressible third grade fluids equations to the solutions of the incompressible third grade fluids equations, we assume that $u_{0}$ is given as in Problem 1.

For given $\epsilon \in(0,1]$, we consider the following initialboundary value problem.

For any given $u_{0} \in H$, set $Q=\Omega \times(0, T)$. Find $u_{\epsilon}: \bar{Q} \rightarrow$ $\mathbb{R}^{3}$ and $p_{\epsilon}: \bar{Q} \rightarrow \mathbb{R}$ such that

$$
\begin{gathered}
\frac{\partial u_{\epsilon}}{\partial t}+v \Delta u_{\epsilon}+\left(u_{\epsilon} \cdot \nabla\right) u_{\epsilon}+\frac{1}{2}\left(\operatorname{div} u_{\epsilon}\right) u_{\epsilon}+J\left(u_{\epsilon}\right) \\
+\beta K(u)+\nabla p_{\epsilon}=0, \\
\epsilon \frac{\partial p_{\epsilon}}{\partial t}+\operatorname{div} u_{\epsilon}=0, \quad \text { in } Q, \\
\left.u_{\epsilon}\right|_{\partial \Omega}=0, \\
u_{\epsilon}(x, 0)=u_{0}, \quad x \in \Omega, \\
p_{\epsilon}(x, 0)=p_{0}, \quad x \in \Omega,
\end{gathered}
$$

where the function $p_{0}$ (not appearing in (5)) is independent of $\epsilon$ and

$$
p_{0} \in L^{2}(\Omega)
$$

Remark 5. Equation (29) contains the term $(1 / 2)\left(\operatorname{div} u_{\epsilon}\right) u_{\epsilon}$ which does not appear in (5). This is a stabilization term which corresponds to the substitution of the trilinear form $\bar{b}$ for the form $b$ (see (14)), where the trilinear form $\bar{b}$ is given by

$$
\begin{array}{r}
\bar{b}(u, v, w)=\frac{1}{2}[b(u, v, w)-b(u, w, v)], \\
\forall u, v, w \in \mathbb{H}_{0}^{1}(\Omega) .
\end{array}
$$

In fact, if $\operatorname{div} u \neq 0$, then $b(u, u, u) \neq 0$. But $\bar{b}(u, v, v)=$ $0, \forall u, v \in \mathbb{H}_{0}^{1}(\Omega)$. Equations (29)-(30) are the constructed slightly compressible third grade fluids equations. This artificial compressibility method has been used by Zhao and You [15] and Zhao [16] to approximate the incompressible convective Brinkman-Forchheimer equations and a class of incompressible non-Newtonian fluids equations.

We first consider the classical solution $\left\{u_{\epsilon}, p_{\epsilon}\right\}$ of (29)(33). Assume that $u_{\epsilon} \in \mathscr{C}^{2}(\bar{Q})$ and $p_{\epsilon} \in \mathscr{C}^{1}(\bar{Q})$. Then, for any $\phi \in \mathscr{C}_{0}^{\infty}(\Omega) \times \mathscr{C}_{0}^{\infty}(\Omega) \times \mathscr{C}_{0}^{\infty}(\Omega)$ and $q \in \mathscr{C}_{0}^{\infty}(\Omega)$, multiplying (29) by $\phi$ and (30) by $q$, we obtain

$$
\begin{gathered}
\frac{\mathrm{d}}{\mathrm{d} t}\left(u_{\epsilon}, \phi\right)+v\left(\nabla u_{\epsilon}, \nabla \phi\right)+\bar{b}\left(u_{\epsilon}, u_{\epsilon}, \phi\right) \\
+\alpha\left(A^{2}\left(u_{\epsilon}\right), \nabla \phi\right)+\beta\left(\left|A\left(u_{\epsilon}\right)\right|^{2} A\left(u_{\epsilon}\right), \nabla \phi\right) \\
+\left(\nabla p_{\epsilon}, \phi\right)=0, \\
\epsilon \frac{\mathrm{d}}{\mathrm{d} t}\left(p_{\epsilon}, q\right)+\left(\operatorname{div} u_{\epsilon}, q\right)=0 .
\end{gathered}
$$

The above two equalities are still valid by a continuity argument for any $\phi \in \mathbb{W}_{0}^{1,4}(\Omega)$ and any $q \in L^{2}(\Omega)$.

Let $\bar{b}$ be defined by (35) and set $\bar{B}(u)=\bar{B}(u, u)$ via

$$
\langle\bar{B}(u), v\rangle=\bar{b}(u, u, v), \quad \forall u, v \in \mathbb{H}_{0}^{1}(\Omega) .
$$

Then, like $B(u)$, the operator $\bar{B}(u)$ is continuous on $\mathbb{M}_{0}^{1}(\Omega)$.

Lemma 6. If $u \in L^{\infty}\left(0, T ; \mathbb{L}^{2}(\Omega)\right) \cap L^{2}\left(0, T ; \mathbb{H}_{0}^{1}(\Omega)\right) \cap$ $L^{4}\left(0, T ; \mathbb{W}_{0}^{1,4}(\Omega)\right)$, then

$$
\begin{gathered}
t \longmapsto \mathscr{L} u(t) \in L^{2}\left(0, T ; \mathbb{H}^{-1}(\Omega)\right) \\
t \longmapsto \bar{B}(u(t)) \in L^{4 / 3}\left(0, T ; \mathbb{H}^{-1}(\Omega)\right) \\
t \longmapsto-\alpha \operatorname{div}\left(A^{2}(u(t))\right) \in L^{2}\left(0, T ; \mathbb{H}^{-1}(\Omega)\right) \\
t \longmapsto-\operatorname{div}\left(|A(u(t))|^{2} A(u(t))\right) \in L^{4 / 3}\left(0, T ; \mathbb{W}^{-1,4 / 3}(\Omega)\right) .
\end{gathered}
$$

Proof. For almost all $t \in[0, T], \mathscr{L} u(t), \bar{B}(u(t))$, and $-\alpha \operatorname{div}\left(A^{2}(u(t))\right)$ are elements of $\mathbb{H}^{-1}(\Omega)$, and $-\operatorname{div}\left(|A(u(t))|^{2} A(u(t))\right)$ is an element of $\mathbb{W}^{-1,4 / 3}(\Omega)$, and the measurability of the functions $t \mapsto \mathscr{L} u(t), t \mapsto \bar{B}(u(t))$, $t \mapsto-\alpha \operatorname{div}\left(A^{2}(u(t))\right)$, and $t \mapsto-\operatorname{div}\left(|A(u(t))|^{2} A(u(t))\right)$ is easy to check. Now for any $\phi \in \mathbb{M}_{0}^{1}(\Omega)$, we have

$$
|\langle\mathscr{L} u(t), \phi\rangle|=|(\nabla u(t), \nabla \phi)| \leq c\|u(t)\|_{\mathbb{\mathbb { O } _ { 0 } ^ { 1 }}(\Omega)}\|\phi\|_{\mathbb{\boxplus}_{0}^{1}(\Omega)} .
$$

By Hölder and Gagliardo-Nirenberg inequalities and the embedding $\mathbb{T}_{0}^{1}(\Omega) \hookrightarrow \mathbb{L}^{6}(\Omega)$, we have

$$
\begin{aligned}
& |\langle\bar{B}(u), \phi\rangle| \\
& =\frac{1}{2}|[b(u, u, \phi)-b(u, \phi, u)]| \\
& \leq \frac{1}{2} \sum_{i, j=1}^{3}\left(\int_{\Omega}\left|u_{i} \frac{\partial u_{j}}{\partial x_{i}} \phi_{j}\right| \mathrm{d} x+\int_{\Omega}\left|u_{i} \frac{\partial \phi_{j}}{\partial x_{i}} u_{j}\right| \mathrm{d} x\right) \\
& \leq c\left(\|u\|_{\mathbb{L}^{3}(\Omega)}\|\nabla u\|\|\phi\|_{\mathbb{L}^{6}(\Omega)}+\|u\|_{\mathbb{L}^{4}(\Omega)}^{2}\|\nabla \phi\|\right) \\
& \leq c\|u\|^{1 / 2}\|u\|_{\mathbb{H}_{0}^{1}(\Omega)}^{3 / 2}\|\phi\|_{\mathbb{R}_{0}^{1}(\Omega)},
\end{aligned}
$$




$$
\begin{aligned}
& |\langle J(u), \phi\rangle| \\
& \quad=\left|\alpha \int_{\Omega} A^{2}(u) \times \nabla \phi \mathrm{d} x\right| \leq c\|A(u(t))\|_{\mathbb{L}^{4}(\Omega)}^{2}\|\phi\|_{\mathbb{1}_{0}^{1}(\Omega)} .
\end{aligned}
$$

Similarly, for any $\phi \in \mathbb{W}_{0}^{1,4}(\Omega)$, we have

$$
\begin{aligned}
|\langle K(u), \phi\rangle| & =\left.\left|\int_{\Omega}\right| A(u)\right|^{2} A(u) \times \nabla \phi \mathrm{d} x \mid \\
& \leq c\|A(u)\|_{\mathbb{L}^{4}(\Omega)}^{3}\|\phi\|_{\mathbb{W}_{0}^{1,4}(\Omega)} .
\end{aligned}
$$

From (42)-(44), we conclude that

$$
\begin{gathered}
\|\mathscr{L} u(t)\|_{\mathbb{Q}^{-1}(\Omega)} \leq c\|u(t)\|_{\mathbb{Q}_{0}^{1}(\Omega)}, \\
\|\bar{B}(u(t))\|_{\mathbb{Q}^{-1}(\Omega)} \leq c\|u(t)\|^{1 / 2}\|u(t)\|_{\mathbb{Q}_{0}^{1}(\Omega)}^{3 / 2}, \\
\|J(u(t))\|_{\mathbb{Q}^{-1}(\Omega)} \leq c\|A(u(t))\|_{\mathbb{Q}^{4}(\Omega)}^{2} \leq c\|u(t)\|_{\mathbb{W}_{0}^{1,4}(\Omega)}^{2}, \\
\|K(u(t))\|_{\mathbb{W}^{-1,4 / 3}(\Omega)} \leq c\|A(u(t))\|_{\mathbb{R}^{4}(\Omega)}^{3} \leq c\|u(t)\|_{\mathbb{W}_{0}^{1,4}(\Omega)}^{3} .
\end{gathered}
$$

Therefore,

$$
\begin{gathered}
\int_{0}^{T}\|\mathscr{L} u(t)\|_{\mathbb{Q}^{-1}(\Omega)}^{2} \mathrm{~d} t \leq c \int_{0}^{T}\|u(t)\|_{\mathbb{Q}_{0}^{1}(\Omega)}^{2} \mathrm{~d} t, \\
\int_{0}^{T}\|\bar{B}(u(t))\|_{\mathbb{Q}^{-1}(\Omega)}^{4 / 3} \mathrm{~d} t \\
\leq c \int_{0}^{T}\|u(t)\|^{2 / 3}\|u(t)\|_{\mathbb{\boxplus}_{0}^{1}(\Omega)}^{2} \mathrm{~d} t \\
\leq c\|u(t)\|_{L^{\infty}\left(0, T ; \mathbb{L}^{2}(\Omega)\right)}^{2 / 3} \int_{0}^{T}\|u(t)\|_{\mathbb{\boxplus}_{0}^{1}(\Omega)}^{2} \mathrm{~d} t, \\
\int_{0}^{T}\|J(u(t))\|_{\mathbb{Q}^{-1}(\Omega)}^{2} \mathrm{~d} t \leq c \int_{0}^{T}\|u(t)\|_{\mathbb{W}_{0}^{1,4}(\Omega)}^{4} \mathrm{~d} t . \\
\int_{0}^{T}\|K(u(t))\|_{\mathbb{W}^{-1,4 / 3}(\Omega)}^{4 / 3} \mathrm{~d} t \leq c \int_{0}^{T}\|u(t)\|_{\mathbb{W}_{0}^{1,4}(\Omega)}^{4} \mathrm{~d} t .
\end{gathered}
$$

Equations (46)-(49) give (38)-(41), respectively. The proof is complete.

Now if $u_{\epsilon} \in L^{\infty}\left(0, T ; \mathbb{L}^{2}(\Omega)\right) \cap L^{2}\left(0, T ; \mathbb{H}_{0}^{1}(\Omega)\right) \cap$ $L^{4}\left(0, T ; \mathbb{W}_{0}^{1,4}(\Omega)\right)$ and $p_{\epsilon} \in L^{2}\left(0, T ; L^{2}(\Omega)\right)$ satisfy $(29)$ and (30) in the distribution sense, then, for any $\phi \in \mathbb{W}_{0}^{1,4}(\Omega)$, we have

$$
\frac{\mathrm{d}}{\mathrm{d} t}\left(u_{\epsilon}, \phi\right)=\left\langle-v \mathscr{L} u_{\epsilon}-\bar{B}\left(u_{\epsilon}\right)-J\left(u_{\epsilon}\right)-\beta K\left(u_{\epsilon}\right)-\nabla p_{\epsilon}, \phi\right\rangle .
$$

Since $p_{\epsilon} \in L^{2}\left(0, T ; L^{2}(\Omega)\right)$, we see from Lemma 6 that

$$
\begin{aligned}
u_{\epsilon}^{\prime} & \doteq \frac{\partial u_{\epsilon}}{\partial t} \\
& =-v \mathscr{L} u_{\epsilon}-\bar{B}\left(u_{\epsilon}\right)-J\left(u_{\epsilon}\right)-\beta K\left(u_{\epsilon}\right)-\nabla p_{\epsilon} \\
& \in L^{4 / 3}\left(0, T ; \mathbb{W}^{-1,4 / 3}(\Omega)\right) .
\end{aligned}
$$

Analogously,

$$
p_{\epsilon}^{\prime} \doteq \frac{\partial p_{\epsilon}}{\partial t} \in L^{2}\left(0, T ; L^{2}(\Omega)\right)
$$

The above analysis leads to the following weak formulation of the problem described by (29)-(33).

Problem 7. Let $\epsilon \in(0,1]$ be fixed. For any given $u_{0} \in H$ and $p_{0} \in L^{2}(\Omega)$, find $u_{\epsilon}$ and $p_{\epsilon}$ such that

$$
\begin{gathered}
u_{\epsilon} \in L^{\infty}\left(0, T ; \mathbb{L}^{2}(\Omega)\right) \\
\cap L^{2}\left(0, T ; \mathbb{H}_{0}^{1}(\Omega)\right) \\
\cap L^{4}\left(0, T ; \mathbb{W}_{0}^{1,4}(\Omega)\right), \\
u_{\epsilon}^{\prime} \in L^{4 / 3}\left(0, T ; \mathbb{W}^{-1,4 / 3}(\Omega)\right), \\
p_{\epsilon} \in L^{2}\left(0, T ; L^{2}(\Omega)\right), \quad p_{\epsilon}^{\prime} \in L^{2}\left(0, T ; L^{2}(\Omega)\right), \\
u_{\epsilon}^{\prime}+v \mathscr{L} u_{\epsilon}+\bar{B}\left(u_{\epsilon}\right)+J\left(u_{\epsilon}\right)+\beta K\left(u_{\epsilon}\right)+\nabla p_{\epsilon}=0, \\
\text { in } \mathscr{D}^{\prime}\left(0, T ; \mathbb{W}^{-1,4 / 3}(\Omega)\right), \\
\epsilon p_{\epsilon}^{\prime}+\operatorname{div} u_{\epsilon}=0, \quad \text { in } \mathscr{D}^{\prime}\left(0, T ; L^{2}(\Omega)\right), \\
u_{\epsilon}(x, 0)=u_{0}, \quad p_{\epsilon}(x, 0)=p_{0} .
\end{gathered}
$$

The rest of this section is devoted to prove the existence of solutions of Problem 7. To this end, we need the compactness theorem (see [13]) involving fractional derivatives in time variable $t$.

Assume $X_{0}, X$, and $X_{1}$ are Hilbert spaces with

$$
X_{0} \hookrightarrow X \hookrightarrow X_{1},
$$

the embedding being continuous and

$$
X_{0} \hookrightarrow X \text { is compact. }
$$

Let $\psi(t)$ be a function from $\mathbb{R}$ to $X_{1}$; we denote by $\mathscr{F}[\psi(t)]=$ $\widehat{\psi}(\tau)$ its Fourier transform:

$$
\widehat{\psi}(\tau)=\int_{-\infty}^{+\infty} \psi(t) \exp (-2 \pi i t \tau) \mathrm{d} t
$$

The derivative in $t$ of order $\gamma$ is the inverse Fourier transform of $(2 i \pi \tau)^{\gamma} \widehat{\psi}(\tau)$; that is,

$$
\widehat{D_{t}^{\gamma} \psi(t)}=(2 i \pi \tau)^{\gamma} \widehat{\psi}(\tau) .
$$

For given $\gamma>0$, define the space

$$
\begin{aligned}
M^{\gamma} & =M^{\gamma}\left(\mathbb{R} ; X_{0}, X_{1}\right) \\
& =\left\{\psi \in L^{2}\left(\mathbb{R} ; X_{0}\right), D_{t}^{\gamma} \psi \in L^{2}\left(\mathbb{R} ; X_{1}\right)\right\} .
\end{aligned}
$$

Then $M^{\gamma}$ is a Hilbert space with the norm

$$
\|\psi\|_{M^{\gamma}}=\left\{\|\psi\|_{L^{2}\left(\mathbb{R} ; X_{0}\right)}^{2}+\left\||\tau|^{\gamma} \widehat{\psi}(\tau)\right\|_{L^{2}\left(\mathbb{R} ; X_{1}\right)}^{2}\right\}^{1 / 2} .
$$


For any set $K \subset \mathbb{R}$, the subspace $M_{K}^{\gamma}$ of $M^{\gamma}$ is defined as the set of functions $u \in M^{\gamma}$ with support contained in $K$ :

$$
M_{K}^{\gamma}=M_{K}^{\gamma}\left(\mathbb{R} ; X_{0}, X_{1}\right)=\left\{\psi \in M^{\gamma}, \operatorname{supp} \psi \subseteq K\right\} .
$$

Lemma 8 (see [13]). Assume $X_{0}, X$, and $X_{1}$ are Hilbert spaces satisfying (59) and (60). Then, for any bounded set $K$ and $\forall \gamma>$ 0 , the following compact embedding holds:

$$
M_{K}^{\gamma}\left(\mathbb{R} ; X_{0}, X_{1}\right) \hookrightarrow L^{2}(\mathbb{R} ; X) .
$$

Theorem 9. Let $\epsilon \in(0,1]$ be fixed. For any given $u_{0} \in H$ and $p_{0} \in L^{2}(\Omega)$, Problem 7 possesses a unique solution $\left\{u_{\epsilon}, p_{\epsilon}\right\}$.

Proof. We will use the Faedo-Galerkin method to prove the existence of solutions for Problem 7. Consider an orthonormal basis of $\mathbb{H}_{0}^{1}(\Omega)$ constituted of elements $w_{n} \epsilon$ $\mathscr{C}_{0}^{\infty}(\Omega) \times \mathscr{C}_{0}^{\infty}(\Omega) \times \mathscr{C}_{0}^{\infty}(\Omega)$ and an orthonormal basis of $L^{2}(\Omega)$ constituted of elements $v_{n} \in \mathscr{C}_{0}^{\infty}(\Omega), n=1,2, \ldots$.

For each positive integer $n$, we define the approximate solution of Problem 7 by

$$
u_{\epsilon n}(t)=\sum_{k=1}^{n} \xi_{k n}(t) w_{k}, \quad p_{\epsilon n}(t)=\sum_{m=1}^{n} \zeta_{m n}(t) v_{m},
$$

which satisfies

$$
\begin{aligned}
& \left(u_{\epsilon n}^{\prime}(t), w_{k}\right)+v\left(\nabla u_{\epsilon n}(t), \nabla w_{k}\right) \\
& \quad+\bar{b}\left(u_{\epsilon n}(t), u_{\epsilon n}(t), w_{k}\right) \\
& \quad+\alpha\left(A^{2}\left(u_{\epsilon n}(t)\right), \nabla w_{k}\right) \\
& \quad+\beta\left(\left|A\left(u_{\epsilon n}(t)\right)\right|^{2} A\left(u_{\epsilon n}(t)\right), \nabla w_{k}\right) \\
& =-\left(\nabla p_{\epsilon n}(t), w_{k}\right), \quad k=1,2, \ldots, n, \\
& \epsilon\left(p_{\epsilon n}^{\prime}(t), v_{m}\right)+\left(\operatorname{div} u_{\epsilon n}(t), v_{m}\right)=0, \\
& m=1,2, \ldots, n,
\end{aligned}
$$

and the initial conditions

$$
u_{\epsilon n}(0)=u_{0 n}, \quad p_{\epsilon n}(0)=p_{0 n},
$$

where $u_{0 n}$ and $p_{0 n}$ are the projections of $u_{0}$ and $p_{0}$ from the spaces $H$ and $L^{2}(\Omega)$ to the spaces $\operatorname{span}\left\{w_{1}, w_{2}, \ldots, w_{n}\right\}$ and $\operatorname{span}\left\{v_{1}, v_{2}, \ldots, v_{n}\right\}$, respectively.

Equations (68)-(70) form a Cauchy problem of firstorder nonlinear ordinary differential equations (ODE) in $t$ for the functions $\xi_{1 n}(t), \xi_{2 n}(t), \ldots, \xi_{n n}(t)$ and $\zeta_{1 n}(t), \zeta_{2 n}(t)$, $\ldots, \zeta_{n n}(t)$ with the following initial conditions:

$$
\begin{gathered}
\xi_{k n}(0)=\text { the } k \text { th component of } u_{0 n}, \quad k=1,2, \ldots, n, \\
\zeta_{m n}(0)=\text { the } m \text { th component of } p_{0 n}, \quad m=1,2, \ldots, n .
\end{gathered}
$$

By the standard theory of ODE, we have the existence of a solution defined on $\left[0, t_{n}\right), 0<t_{n} \leq T$. Moreover, if $t_{n}<T$, then we have the following blow-up criteria $\lim _{t \rightarrow t_{n}^{-}}\left\|u_{\epsilon n}(t)\right\|=+\infty$ and $\lim _{t \rightarrow t_{n}^{-}}\left\|p_{\epsilon n}(t)\right\|=+\infty$.

Multiplying (68) by $\xi_{k n}(t)(k=1,2, \ldots, n)$ and (69) by $\zeta_{m n}(t)(m=1,2, \ldots, n)$ and then adding these resulting equalities, we obtain

$$
\begin{aligned}
\frac{1}{2} \frac{\mathrm{d}}{\mathrm{d} t}\left\|u_{\epsilon n}\right\|^{2}+v\left\|\nabla u_{\epsilon n}\right\|^{2}+\bar{b}\left(u_{\epsilon n}, u_{\epsilon n}, u_{\epsilon n}\right) \\
+\alpha \int_{\Omega} A^{2}\left(u_{\epsilon n}\right) \times \nabla u_{\epsilon n} \mathrm{~d} x \\
+\beta \int_{\Omega}\left|A\left(u_{\epsilon n}\right)\right|^{2} A\left(u_{\epsilon}\right) \times \nabla u_{\epsilon n} \mathrm{~d} x+\left(\nabla p_{\epsilon n}, u_{\epsilon n}\right) \\
+\frac{1}{2} \frac{\mathrm{d}}{\mathrm{d} t} \epsilon\left\|p_{\epsilon n}\right\|^{2}+\left(\operatorname{div} u_{\epsilon n}, p_{\epsilon n}\right)=0 .
\end{aligned}
$$

Set $\sigma_{0}=1-\sqrt{\alpha^{2} / 2 \nu \beta}>0$. Using integration by parts and Hölder inequality, we have

$$
\begin{aligned}
& \left|\alpha \int_{\Omega} A^{2}\left(u_{\epsilon n}\right) \times \nabla u_{\epsilon n} \mathrm{~d} x\right| \\
& \quad \leq|\alpha|\left\|A\left(u_{\epsilon n}\right)\right\|_{\mathbb{L}^{4}(\Omega)}^{2}\left\|\nabla u_{\epsilon n}\right\| \\
& \quad \leq \nu\left(1-\sigma_{0}\right)\left\|\nabla u_{\epsilon n}\right\|^{2}+\frac{\beta\left(1-\sigma_{0}\right)}{2}\left\|A\left(u_{\epsilon n}\right)\right\|_{\mathbb{L}^{4}(\Omega)}^{4} .
\end{aligned}
$$

Also integrating by parts, using the fact that $A\left(u_{\epsilon n}\right)$ is a symmetric matrix, we get

$$
\beta \int_{\Omega}\left|A\left(u_{\epsilon n}\right)\right|^{2} A\left(u_{\epsilon}\right) \times \nabla u_{\epsilon n} \mathrm{~d} x=\frac{\beta}{2}\left\|A\left(u_{\epsilon n}\right)\right\|_{\mathbb{L}^{4}(\Omega)}^{4} .
$$

Since $\bar{b}\left(u_{\epsilon n}, u_{\epsilon n}, u_{\epsilon n}\right)=0$ and $\left.u_{\epsilon n}\right|_{\partial \Omega}=0$, we have

$$
\left(\nabla p_{\epsilon n}, u_{\epsilon n}\right)+\left(\operatorname{div} u_{\epsilon n}, p_{\epsilon n}\right)=0 .
$$

By combining (72)-(75), we deduce that

$$
\begin{gathered}
\frac{\mathrm{d}}{\mathrm{d} t}\left(\left\|u_{\epsilon n}\right\|^{2}+\epsilon\left\|p_{\epsilon n}\right\|^{2}\right)+2 \nu \sigma_{0}\left\|\nabla u_{\epsilon n}\right\|^{2} \\
+\beta \sigma_{0}\left\|A\left(u_{\epsilon n}\right)\right\|_{\mathbb{L}^{4}(\Omega)}^{4} \leq 0 .
\end{gathered}
$$

Integrating (76) in $t$ from 0 to $s\left(\leq t_{n}\right)$ shows that

$$
\begin{aligned}
& \left\|u_{\epsilon n}\right\|^{2}+\epsilon\left\|p_{\epsilon n}\right\|^{2}+2 \nu \sigma_{0} \int_{0}^{s}\left\|\nabla u_{\epsilon n}\right\|^{2} \mathrm{~d} t \\
& +\beta \sigma_{0} \int_{0}^{s}\left\|A\left(u_{\epsilon n}\right)\right\|_{\mathbb{L}^{4}(\Omega)}^{4} \mathrm{~d} t \\
& \leq\left\|u_{0}\right\|^{2}+\epsilon\left\|p_{0}\right\|^{2} \leq\left\|u_{0}\right\|^{2}+\left\|p_{0}\right\|^{2} \doteq d .
\end{aligned}
$$

Thus $t_{n}=T$ and

$$
\begin{gathered}
\sup _{t \in[0, T]}\left(\left\|u_{\epsilon n}(t)\right\|^{2}+\epsilon\left\|p_{\epsilon n}(t)\right\|^{2}\right) \leq d, \\
\int_{0}^{T}\left\|\nabla u_{\epsilon n}\right\|^{2} \mathrm{~d} t \leq \frac{d}{2 \nu \sigma_{0}}, \\
\int_{0}^{T}\left\|A\left(u_{\epsilon n}\right)\right\|_{\mathbb{L}^{4}(\Omega)}^{4} \mathrm{~d} t \leq \frac{d}{\beta \sigma_{0}} .
\end{gathered}
$$


We now need an estimate of the fractional derivative in time $t$ of $u_{\epsilon n}(t)$ to pass to the limit in the nonlinear terms in (68). Set

$$
\begin{aligned}
\psi_{\epsilon n}(t)= & -v \mathscr{L} u_{\epsilon n}(t)-\bar{B}\left(u_{\epsilon n}(t)\right) \\
& -J\left(u_{\epsilon n}(t)\right)-\beta K\left(u_{\epsilon n}(t)\right) .
\end{aligned}
$$

We write the relations (68) and (69) as

$$
\begin{gathered}
\left(u_{\epsilon n}^{\prime}(t), w_{k}\right)+\left(\nabla p_{\epsilon n}(t), w_{k}\right)=\left(\psi_{\epsilon n}(t), w_{k}\right), \\
k=1,2, \ldots, n, \\
\epsilon\left(p_{\epsilon n}^{\prime}(t), v_{m}\right)+\left(\operatorname{div} u_{\epsilon n}(t), v_{m}\right)=0, \\
m=1,2, \ldots, n .
\end{gathered}
$$

Set

$$
\begin{aligned}
& \tilde{u}_{\epsilon n}(t)= \begin{cases}u_{e n}(t), & t \in[0, T], \\
0, & t \in \mathbb{R} \backslash[0, T] .\end{cases} \\
& \tilde{p}_{\epsilon n}(t)= \begin{cases}p_{\epsilon n}(t), & t \in[0, T], \\
0, & t \in \mathbb{R} \backslash[0, T] .\end{cases}
\end{aligned}
$$

Then both $\widetilde{u}_{\epsilon n}(t)$ and $\widetilde{p}_{\epsilon n}(t)$ have two discontinuities at $t=0$ and $t=T$. Thus we have on the whole $\mathbb{R}$ the following

$$
\begin{array}{r}
\frac{\mathrm{d}}{\mathrm{d} t}\left(\widetilde{u}_{\epsilon n}(t), w_{k}\right)+\left(\nabla \widetilde{p}_{\epsilon n}(t), w_{k}\right) \\
=\left(\widetilde{\psi}_{\epsilon n}(t), w_{k}\right)+\left(u_{0 n}, w_{k}\right) \delta_{(0)} \\
\quad-\left(u_{\epsilon n}(T), w_{k}\right) \delta_{(T)}, \quad k=1,2, \ldots, n, \\
\frac{\mathrm{d}}{\mathrm{d} t} \epsilon\left(\widetilde{p}_{\epsilon n}(t), v_{m}\right)+\left(\operatorname{div} \widetilde{u}_{\epsilon n}(t), v_{m}\right) \\
=\epsilon\left(p_{0 n}, v_{m}\right) \delta_{(0)}-\epsilon\left(u_{\epsilon n}(T), v_{m}\right) \delta_{(T)}, \\
m=1,2, \ldots, n,
\end{array}
$$

where $\widetilde{\psi}_{\epsilon n}(t)$ is defined similarly with that of $\widetilde{u}_{\epsilon n}(t)$ and $\delta_{(0)}$ and $\delta_{(T)}$ are the Dirac distributions at 0 and $T$, respectively. Taking the Fourier transform on both sides of (84), we get

$$
\begin{array}{r}
2 i \pi \tau\left(\widehat{u}_{\epsilon n}(\tau), w_{k}\right)+\left(\nabla \widehat{p}_{\epsilon n}(\tau), w_{k}\right) \\
=\left(\widehat{\psi}_{\epsilon n}(\tau), w_{k}\right)+\left(u_{0 n}, w_{k}\right) \\
\quad-\left(u_{\epsilon n}(T), w_{k}\right) e^{-2 i \pi \tau T}, \quad k=1, \ldots, n, \\
2 i \pi \tau \epsilon\left(\widehat{p}_{\epsilon n}(\tau), v_{m}\right)+\left(\operatorname{div} \widehat{u}_{\epsilon n}(\tau), v_{m}\right) \\
=\epsilon\left(p_{0 n}, v_{m}\right)-\epsilon\left(u_{\epsilon n}(T), v_{m}\right) e^{-2 i \pi \tau T}, \\
m=1, \ldots, n .
\end{array}
$$

Similar with (83), we can define $\widetilde{\xi}_{k n}(t)$ and $\widetilde{\zeta}_{m n}(t)$ and let $\widehat{\xi}_{k n}(\tau)$ and $\widehat{\zeta}_{m n}(\tau)$ be the Fourier transforms of $\widetilde{\xi}_{k n}(t)$ and $\widetilde{\zeta}_{m n}(t)$, respectively. Multiplying (85) by $\widehat{\xi}_{k n}(\tau)(k=1,2, \ldots$, $n)$ and (86) by $\widehat{\zeta}_{m n}(\tau)(m=1,2, \ldots, n)$ and then adding these resulting equalities, we obtain

$$
\begin{aligned}
2 i \pi \tau & \left(\left\|\widehat{u}_{\epsilon n}(\tau)\right\|^{2}+\epsilon\left\|\widehat{p}_{\epsilon n}(\tau)\right\|^{2}\right) \\
& +\left(\nabla \widehat{p}_{\epsilon n}(\tau), \widehat{u}_{\epsilon n}(\tau)\right)+\left(\operatorname{div} \widehat{u}_{\epsilon n}(\tau), \widehat{p}_{\epsilon n}(\tau)\right) \\
= & \left\langle\widehat{\psi}_{n}(\tau), \widehat{u}_{\epsilon n}(\tau)\right\rangle+\left(u_{0 n}, \widehat{u}_{\epsilon n}(\tau)\right)+\epsilon\left(p_{0 n}, \widehat{p}_{\epsilon n}(\tau)\right) \\
& -\left[\left(u_{\epsilon n}(T), \widehat{u}_{\epsilon n}(\tau)\right)+\epsilon\left(p_{\epsilon n}(T), \widehat{p}_{\epsilon n}(\tau)\right)\right] e^{-2 i \pi \tau T} .
\end{aligned}
$$

Since $\left.\widehat{u}_{\epsilon n}(\tau)\right|_{\partial \Omega}=0$, integration by parts gives

$$
\left(\nabla \widehat{p}_{\epsilon n}(\tau), \widehat{u}_{\epsilon n}(\tau)\right)+\left(\operatorname{div} \widehat{u}_{\epsilon n}(\tau), \widehat{p}_{\epsilon n}(\tau)\right)=0 .
$$

It then follows from (87), (88), and then (78) that

$$
\begin{aligned}
2 \pi|\tau| & \left\|\widehat{u}_{\epsilon n}(\tau)\right\|^{2} \\
\leq & \left|\left\langle\widehat{\psi}_{\epsilon n}(\tau), \widehat{u}_{\epsilon n}(\tau)\right\rangle\right|+\left\|u_{0 n}\right\|\left\|\widehat{u}_{\epsilon n}(\tau)\right\| \\
& +\epsilon\left\|p_{0 n}\right\|\left\|\widehat{p}_{\epsilon n}(\tau)\right\| \\
& +\left\|u_{\epsilon n}(T)\right\|\left\|\widehat{u}_{\epsilon n}(\tau)\right\|+\epsilon\left\|p_{\epsilon n}(T)\right\|\left\|\widehat{p}_{\epsilon n}(\tau)\right\| \\
\leq & \left|\left\langle\widehat{\psi}_{\epsilon n}(\tau), \widehat{u}_{\epsilon n}(\tau)\right\rangle\right| \\
& +2 \sqrt{d}\left(\left\|\widehat{u}_{\epsilon n}(\tau)\right\|+\epsilon\left\|\widehat{p}_{\epsilon n}(\tau)\right\|\right) .
\end{aligned}
$$

We next estimate the term $\left|\left\langle\widehat{\psi}_{e n}(\tau), \widehat{u}_{\epsilon n}(\tau)\right\rangle\right|$. In fact, using the similar derivations as (73) and (74), we get

$$
\begin{aligned}
& \left|\left\langle\widehat{\psi}_{\epsilon n}(\tau), \widehat{u}_{\epsilon n}(\tau)\right\rangle\right| \\
& \leq v\left|\nabla \widehat{u}_{\epsilon n}(\tau) \|^{2}+\right| \bar{b}\left(\widehat{u}_{\epsilon n}(\tau), \widehat{u}_{\epsilon n}(\tau), \widehat{u}_{\epsilon n}(\tau)\right) \mid \\
& \quad+\left|\alpha \int_{\Omega} \operatorname{div}\left(A^{2}\left(\widehat{u}_{\epsilon n}(\tau)\right) \widehat{u}_{\epsilon n}(\tau)\right) \mathrm{d} x\right| \\
& \quad+\left|\beta \int_{\Omega} \operatorname{div}\left(\left|A\left(\widehat{u}_{\epsilon n}(\tau)\right)\right|^{2} A\left(\widehat{u}_{\epsilon n}(\tau)\right) \widehat{u}_{\epsilon n}(\tau)\right) \mathrm{d} x\right| \\
& \leq\left(\nu+\frac{\alpha}{2}\right)\left\|\nabla \widehat{u}_{\epsilon n}(\tau)\right\|^{2}+\frac{\alpha+\beta}{2}\left\|A\left(\widehat{u}_{\epsilon n}(\tau)\right)\right\|_{\mathbb{L}^{4}(\Omega)}^{4} .
\end{aligned}
$$

Inserting (90) into (89) gives

$$
\begin{aligned}
& 2 \pi|\tau|\left\|\widehat{u}_{\epsilon n}(\tau)\right\|^{2} \\
& \leq\left(\nu+\frac{\alpha}{2}\right)\left\|\nabla \widehat{u}_{\epsilon n}(\tau)\right\|^{2}+\frac{\alpha+\beta}{2}\left\|A\left(\widehat{u}_{\epsilon n}(\tau)\right)\right\|_{\mathbb{L}^{4}(\Omega)}^{4} \\
& \quad+2 \sqrt{d}\left(\left\|\widehat{u}_{\epsilon n}(\tau)\right\|+\epsilon\left\|\widehat{p}_{\epsilon n}(\tau)\right\|\right) .
\end{aligned}
$$

For some fixed $\gamma \in(0,1 / 4)$, we have $|\tau|^{2 \gamma} \leq(2 \gamma+1)((1+$ $\left.|\tau|) /\left(1+|\tau|^{1-2 \gamma}\right)\right), \forall \tau \in \mathbb{R}$. Thus

$$
\begin{aligned}
& \int_{-\infty}^{+\infty}|\tau|^{2 \gamma}\left\|\widehat{u}_{\epsilon n}(\tau)\right\|^{2} \mathrm{~d} \tau \\
& \quad \leq(2 \gamma+1) \int_{-\infty}^{+\infty} \frac{\left\|\widehat{u}_{\epsilon n}(\tau)\right\|^{2}}{1+|\tau|^{1-2 \gamma}} \mathrm{d} \tau
\end{aligned}
$$




$$
\begin{aligned}
& +(2 \gamma+1) \int_{-\infty}^{+\infty} \frac{|\tau|\left\|\widehat{u}_{\epsilon n}(\tau)\right\|^{2}}{1+|\tau|^{1-2 \gamma}} \mathrm{d} \tau \\
\leq & \underbrace{(2 \gamma+1) \int_{-\infty}^{+\infty}\left\|\widehat{u}_{\epsilon n}(\tau)\right\|^{2} \mathrm{~d} \tau}_{I_{1}} \\
& +\underbrace{(2 \gamma+1) \int_{-\infty}^{+\infty} \frac{|\tau|\left\|\widehat{u}_{\epsilon n}(\tau)\right\|^{2}}{1+|\tau|^{1-2 \gamma}} \mathrm{d} \tau}_{I_{2}} .
\end{aligned}
$$

Employing Parseval equality, Poincaré inequality, and (79), we obtain

$$
\begin{aligned}
I_{1} & =(2 \gamma+1) \int_{-\infty}^{+\infty}\left\|\tilde{u}_{\epsilon n}\right\|^{2} \mathrm{~d} t \\
& =(2 \gamma+1) \int_{0}^{T}\left\|u_{\epsilon n}\right\|^{2} \mathrm{~d} t \leq c\left(\gamma, v, \sigma_{0}, d\right) .
\end{aligned}
$$

By (91) and the fact that $2 \gamma+1<2$, we obtain

$$
\begin{aligned}
I_{2} \leq & \underbrace{\frac{\nu+\alpha / 2}{\pi} \int_{-\infty}^{+\infty}\left\|\nabla \widehat{u}_{\epsilon n}(\tau)\right\|^{2} \mathrm{~d} \tau+\frac{\alpha+\beta}{2 \pi} \int_{-\infty}^{+\infty}\left\|A\left(\widehat{u}_{\epsilon n}(\tau)\right)\right\|_{\mathbb{L}^{4}(\Omega)}^{4} \mathrm{~d} \tau}_{I_{3}} \\
& +\underbrace{\frac{2 \sqrt{d}}{\pi} \int_{-\infty}^{+\infty} \frac{\left\|\widehat{u}_{\epsilon n}(\tau)\right\|+\epsilon\left\|\widehat{p}_{\epsilon n}(\tau)\right\|}{1+|\tau|^{1-2 \gamma}} \mathrm{d} \tau}_{I_{4}} .
\end{aligned}
$$

Equations (79), (80), and Parseval equality imply

$$
I_{3} \leq \frac{\nu+\alpha / 2}{\pi} \frac{d}{2 \nu \sigma_{0}}+\frac{\alpha+\beta}{2 \pi} \frac{d}{\beta \sigma_{0}} .
$$

Using Hölder inequality, (78), and the convergence of the infinite integral

$$
\left(\int_{-\infty}^{+\infty} \frac{\mathrm{d} \tau}{\left(1+|\tau|^{1-2 \gamma}\right)^{2}}\right)^{1 / 2}, \quad \text { for some } \gamma \in\left(0, \frac{1}{4}\right)
$$

we obtain

$$
\begin{aligned}
I_{4} \leq & \frac{4 \sqrt{d}}{\pi}\left(\int_{-\infty}^{+\infty} \frac{\mathrm{d} \tau}{\left(1+|\tau|^{1-2 \gamma}\right)^{2}}\right)^{1 / 2} \\
& \cdot\left(\int_{-\infty}^{+\infty}\left(\left\|\widetilde{u}_{\epsilon n}(t)\right\|^{2}+\epsilon\left\|\tilde{p}_{\epsilon n}(t)\right\|^{2}\right) \mathrm{d} t\right)^{1 / 2} \\
= & \frac{4 \sqrt{d}}{\pi}\left(\int_{-\infty}^{+\infty} \frac{\mathrm{d} \tau}{\left(1+|\tau|^{1-2 \gamma}\right)^{2}}\right)^{1 / 2} \\
& \cdot\left(\int_{0}^{T}\left(\left\|u_{\epsilon n}(t)\right\|^{2}+\epsilon\left\|p_{\epsilon n}(t)\right\|^{2}\right) \mathrm{d} t\right)^{1 / 2} \\
\leq & c(\gamma, d, T) .
\end{aligned}
$$

Equations (92)-(97) give

$$
\int_{-\infty}^{+\infty}|\tau|^{2 \gamma}\left\|\widehat{u}_{\epsilon n}(\tau)\right\|^{2} \mathrm{~d} \tau \leq c \quad(\text { in dependent of } n \text { and } \epsilon) .
$$

Equations (78)-(80), as well as Lemma 8 and (98), imply that we can extract a subsequence (still denoted by $\left\{u_{\epsilon n}\right\}_{n=1}^{\infty}$ ) of $\left\{u_{\epsilon n}\right\}_{n=1}^{\infty}$ such that

$$
\begin{aligned}
& u_{\epsilon n} \stackrel{\text { weak-star }}{\longrightarrow} u_{\epsilon} \quad \text { in } L^{\infty}\left(0, T ; \mathbb{L}^{2}(\Omega)\right), \\
& u_{\epsilon n} \stackrel{\text { weakly }}{\longrightarrow} u_{\epsilon} \quad \text { in } L^{2}\left(0, T ; \mathbb{T}_{0}^{1}(\Omega)\right), \\
& u_{\epsilon n} \stackrel{\text { strongly }}{\longrightarrow} u_{\epsilon} \quad \text { in } L^{2}\left(0, T ; \mathbb{L}^{2}(\Omega)\right), \\
& u_{\epsilon n} \stackrel{\text { weakly }}{\longrightarrow} u_{\epsilon} \quad \text { in } L^{4}\left(0, T ; \mathbb{W}_{0}^{1,4}(\Omega)\right), \\
& p_{\epsilon n} \stackrel{\text { weak-star }}{\longrightarrow} p_{\epsilon} \quad \text { in } L^{\infty}\left(0, T ; L^{2}(\Omega)\right) .
\end{aligned}
$$

Taking $\varphi(t) \in \mathscr{C}_{0}^{\infty}(0, T)$ and multiplying (68) (resp., (69)) by $\varphi(t)$, integrating over $(0, T)$, and then integrating the first term by parts, we obtain

$$
\begin{aligned}
-\int_{0}^{T} & \left\langle u_{\epsilon n}(t), w_{k}\right\rangle \varphi^{\prime}(t) \mathrm{d} t \\
& -v \int_{0}^{T}\left(\Delta u_{\epsilon n}(t), w_{k} \varphi(t)\right) \mathrm{d} t \\
& +\underbrace{\int_{0}^{T} \bar{b}\left(u_{\epsilon n}(t), u_{\epsilon n}(t), w_{k} \varphi(t)\right) \mathrm{d} t}_{I_{5}} \\
- & \underbrace{\alpha \int_{0}^{T}\left(\operatorname{div}\left(A^{2}\left(u_{\epsilon n}(t)\right)\right), w_{k} \varphi(t)\right) \mathrm{d} t}_{I_{6}}
\end{aligned}
$$




$$
\begin{gathered}
-\underbrace{\beta \int_{0}^{T}\left(\operatorname{div}\left(\left|A\left(u_{\epsilon n}(t)\right)\right|^{2} A\left(u_{\epsilon n}(t)\right)\right), w_{k} \varphi(t)\right) \mathrm{d} t}_{I_{7}} \\
+\int_{0}^{T}\left(\nabla p_{\epsilon n}(t), w_{k} \varphi(t)\right) \mathrm{d} t=0, \quad k=1,2, \ldots, n, \\
-\epsilon \int_{0}^{T}\left(p_{\epsilon n}(t), v_{m}\right) \varphi^{\prime}(t) \mathrm{d} t \\
+\int_{0}^{T}\left(\operatorname{div} u_{\epsilon n^{\prime}}(t), v_{m} \varphi(t)\right) \mathrm{d} t=0, \quad m=1,2, \ldots, n .
\end{gathered}
$$

We now examine the convergence of the nonlinear terms $I_{5}$, $I_{6}$, and $I_{7}$ in (104). Firstly, consider

$$
\begin{aligned}
2 \mid I_{5} & -\int_{0}^{T} \bar{b}\left(u_{\epsilon}, u_{\epsilon}, w_{k} \varphi(t)\right) \mathrm{d} t \mid \\
\leq & \mid \int_{0}^{T} b\left(u_{\epsilon n}, u_{\epsilon n}, w_{k} \varphi(t)\right) \mathrm{d} t \\
& -\int_{0}^{T} b\left(u_{\epsilon}, u_{\epsilon}, w_{k} \varphi(t)\right) \mathrm{d} t \mid \\
& +\mid \int_{0}^{T} b\left(u_{\epsilon n}, w_{k} \varphi(t), u_{\epsilon n}\right) \mathrm{d} t \\
\leq & \underbrace{\left|\int_{0}^{T} b\left(u_{\epsilon n}-u_{\epsilon}, u_{\epsilon n}, w_{k} \varphi(t)\right) \mathrm{d} t\right|}_{I_{0}} \\
& +\underbrace{\left|\int_{0}^{T} b\left(u_{\epsilon}, w_{k} \varphi(t), u_{\epsilon n}-u_{\epsilon}\right) \mathrm{d} t\right|}_{I_{8}} . \\
& +\left|\int_{0}^{T} b\left(u_{\epsilon}, u_{\epsilon n}-u_{k}, w_{k} \varphi(t), u_{\epsilon}\right) \mathrm{d} t\right| \mathrm{d} t \mid \\
& \underbrace{T}_{I_{10}} b\left(u_{\epsilon n}-u_{\epsilon}, w_{k} \varphi(t), u_{\epsilon n}\right) \mathrm{d} t \mid
\end{aligned}
$$

Using Hölder inequality, (78)-(79), and (101), we get

$$
\begin{aligned}
I_{8} \leq & \sup _{t \in(0, T)}|\varphi(t)| \sup _{x \in \Omega}\left|w_{k}\right| \\
& \cdot \int_{0}^{T}\left\|u_{\epsilon n}-u_{\epsilon}\right\|\left\|\nabla u_{\epsilon n}\right\| \mathrm{d} t \\
\leq c & \left\|u_{\epsilon n}-u_{\epsilon}\right\|_{L^{2}\left(0, T ; \mathbb{L}^{2}(\Omega)\right)}\left\|u_{\epsilon n}\right\|_{L^{2}\left(0, T ; \mathbb{H}_{0}^{1}(\Omega)\right)} \\
& \longrightarrow 0 \text { as } n \longrightarrow \infty .
\end{aligned}
$$

Similarly, consider

$$
\begin{aligned}
& I_{9}=\left|\int_{0}^{T} \sum_{m, j=1}^{3} \int_{\Omega}\left(u_{\epsilon}\right)_{m} \frac{\partial\left(u_{\epsilon n}-u_{\epsilon}\right)_{j}}{\partial x_{m}}\left(\varphi(t) w_{k}\right)_{j} \mathrm{~d} x \mathrm{~d} t\right| \\
& \leq \int_{0}^{T} \sum_{m, j=1}^{3}\left|\int_{\Omega}\left(u_{\epsilon n}-u_{\epsilon}\right)_{j} \frac{\partial\left(u_{\epsilon}\right)_{m}}{\partial x_{m}}\left(\varphi(t) w_{k}\right)_{j}\right| \mathrm{d} x \mathrm{~d} t \\
& +\int_{0}^{T} \sum_{m, j=1}^{3}\left|\int_{\Omega}\left(u_{\epsilon n}-u_{\epsilon}\right)_{j}\left(u_{\epsilon}\right)_{m} \varphi(t) \frac{\partial\left(w_{k}\right)_{j}}{\partial x_{m}}\right| \mathrm{d} x \mathrm{~d} t \\
& \leq \sup _{t \in(0, T)}|\varphi(t)| \sup _{x \in \Omega}\left|w_{k}\right| \int_{0}^{T}\left\|u_{\epsilon n}-u_{\epsilon}\right\|\left\|\nabla u_{\epsilon}\right\| \mathrm{d} t \\
& +\sup _{t \in(0, T)}|\varphi(t)| \sup _{x \in \Omega}\left|\nabla w_{k}\right| \int_{0}^{T}\left\|u_{\epsilon n}-u_{\epsilon}\right\|\left\|u_{\epsilon}\right\| \mathrm{d} t \\
& \leq c\left\|u_{\epsilon n}-u_{\epsilon}\right\|_{L^{2}\left(0, T ; \mathbb{L}^{2}(\Omega)\right)} \\
& \cdot\left(\left\|u_{\epsilon}\right\|_{L^{2}\left(0, T ; \mathbb{H}_{0}^{1}(\Omega)\right)}+\left\|u_{\epsilon}\right\|_{L^{2}\left(0, T ; \mathbb{L}^{2}(\Omega)\right)}\right) \\
& \longrightarrow 0 \text { as } n \longrightarrow \infty \text {, } \\
& I_{10} \leq \sup _{t \in(0, T)}|\varphi(t)| \sup _{x \in \Omega}\left|\nabla w_{k}\right| \int_{0}^{T}\left\|u_{\epsilon n}-u_{\epsilon}\right\|\left\|\nabla u_{\epsilon n}\right\| \mathrm{d} t \\
& \leq c\left\|u_{\epsilon n}-u_{\epsilon}\right\|_{L^{2}\left(0, T ; \mathbb{L}^{2}(\Omega)\right)}\left\|u_{\epsilon n}\right\|_{L^{2}\left(0, T ; \square_{0}^{1}(\Omega)\right)} \\
& \longrightarrow 0 \text { as } n \longrightarrow \infty \\
& I_{11} \leq \sup _{t \in(0, T)}|\varphi(t)| \sup _{x \in \Omega}\left|\nabla w_{k}\right| \int_{0}^{T}\left\|u_{\epsilon n}-u_{\epsilon}\right\|\left\|u_{\epsilon}\right\| \mathrm{d} t \\
& \leq c\left\|u_{\epsilon n}-u_{\epsilon}\right\|_{L^{2}\left(0, T ; \mathbb{L}^{2}(\Omega)\right)}\left\|u_{\epsilon}\right\|_{L^{2}\left(0, T ; \mathbb{L}^{2}(\Omega)\right)} \\
& \longrightarrow 0 \text { as } n \longrightarrow \infty \text {. }
\end{aligned}
$$

Equatuions (106)-(110) imply that

$$
I_{5} \longrightarrow \int_{0}^{T} \bar{b}\left(u_{\epsilon}, u_{\epsilon}, w_{k} \varphi(t)\right) \mathrm{d} t \quad \text { as } n \longrightarrow \infty
$$

Secondly, the following convergence

$$
\begin{aligned}
I_{6}+I_{7} \longrightarrow & \alpha \int_{0}^{T}\left(\operatorname{div}\left(A^{2}\left(u_{\epsilon}(t)\right)\right), w_{k} \varphi(t)\right) \mathrm{d} t \\
& +\beta \int_{0}^{T}\left(\operatorname{div}\left(\left|A\left(u_{\epsilon}(t)\right)\right|^{2} A\left(u_{\epsilon}(t)\right)\right), w_{k} \varphi(t)\right) \mathrm{d} t
\end{aligned}
$$$$
\text { as } n \longrightarrow \infty
$$ 
can be proved by using the same way as that in [7], and we omit the details here. It is not difficult to pass to the limit in the linear terms in (104) and (105). Now for fixed $k=1,2, \ldots, n$ and $m=1,2, \ldots, n$, taking the limit of (104) and (105) as $n \rightarrow$ $\infty$, using (99)-(103) and (111)-(112), we obtain

$$
\begin{aligned}
& -\int_{0}^{T}\left\langle u_{\epsilon}(t), w_{k}\right\rangle \varphi^{\prime}(t) \mathrm{d} t \\
& \quad+\nu \int_{0}^{T}\left(\nabla u_{\epsilon}(t), \varphi(t) \nabla w_{k}\right) \mathrm{d} t \\
& \quad+\underbrace{\int_{0}^{T} \bar{b}\left(u_{\epsilon}(t), u_{\epsilon}(t), w_{k} \varphi(t)\right) \mathrm{d} t}_{I_{12}} \\
& \quad-\alpha \underbrace{\alpha \int_{0}^{T}\left(\operatorname{div}\left(A^{2}\left(u_{\epsilon}(t)\right)\right), w_{k} \varphi(t)\right) \mathrm{d} t}_{I_{13}} \\
& \quad-\beta \underbrace{\int_{0}^{T}\left(\operatorname{div}\left(\left|A\left(u_{\epsilon}(t)\right)\right|^{2} A\left(u_{\epsilon}(t)\right)\right), w_{k} \varphi(t)\right) \mathrm{d} t}_{0} \\
& \quad+\int_{0}^{T}\left(\nabla p_{\epsilon}(t), w_{k} \varphi(t)\right) \mathrm{d} t=0, \quad k=1,2, \ldots, n, \\
& -\epsilon \int_{0}^{T}\left(p_{\epsilon}(t), v_{m}\right) \varphi^{\prime}(t) \mathrm{d} t \\
& \quad+\int_{0}^{T}\left(\operatorname{div} u_{\epsilon}(t), v_{m} \varphi(t)\right) \mathrm{d} t=0, \quad m=1,2, \ldots, n .
\end{aligned}
$$

Since $\Omega \subset \mathbb{R}^{3}$, we have the embeddings $\mathbb{U}_{0}^{1}(\Omega) \hookrightarrow \mathbb{L}^{6}(\Omega) \hookrightarrow$ $\mathbb{L}^{4}(\Omega) \hookrightarrow \mathbb{L}^{18 / 5}(\Omega)$ and use the Hölder inequality to get

$$
\begin{aligned}
I_{12} \leq & \frac{1}{2}\left|\int_{0}^{T} b\left(u_{\epsilon}(t), u_{\epsilon}(t), w_{k} \varphi(t)\right) \mathrm{d} t\right| \\
& +\frac{1}{2}\left|\int_{0}^{T} b\left(u_{\epsilon}(t), w_{k} \varphi(t), u_{\epsilon}(t)\right) \mathrm{d} t\right| \\
\leq & \frac{1}{2} \sup _{t \in(0, T)}|\varphi(t)| \int_{0}^{T}\left\|u_{\epsilon}\right\|_{\mathbb{L}^{4}(\Omega)}\left\|\nabla u_{\epsilon}\right\|\left\|w_{k}\right\|_{\mathbb{L}^{4}(\Omega)} \mathrm{d} t \\
& +\frac{1}{2} \sup _{t \in(0, T)}|\varphi(t)| \int_{0}^{T}\left\|u_{\epsilon}\right\|_{\mathbb{L}^{4}(\Omega)}^{2}\left\|\nabla w_{k}\right\| \mathrm{d} t \\
\leq & c\left\|u_{\epsilon}\right\|_{L^{2}\left(0, T ; \forall \uplus_{0}^{1}(\Omega)\right)}^{2}\left\|w_{k}\right\|_{\mathbb{R}_{0}^{1}(\Omega)} .
\end{aligned}
$$

Using integration by parts, we have

$$
\begin{aligned}
\left|I_{13}\right| & =\left|\int_{0}^{T}\left(A^{2}\left(u_{\epsilon}(t)\right), \varphi(t) \nabla w_{k}\right) \mathrm{d} t\right| \\
& \leq \sup _{t \in(0, T)}|\varphi(t)| \int_{0}^{T}\left\|A^{2}\left(u_{\epsilon}(t)\right)\right\|\left\|\nabla w_{k}\right\| \mathrm{d} t \\
& \leq c\left\|u_{\epsilon}\right\|_{L^{2}\left(0, T ; \mathbb{W}_{0}^{1,4}(\Omega)\right)}^{2}\left\|w_{k}\right\|_{\mathbb{1}_{0}^{1}(\Omega)},
\end{aligned}
$$

$$
\begin{aligned}
\left|I_{14}\right| & =\left|\int_{0}^{T}\left(\left|A\left(u_{\epsilon}(t)\right)\right|^{2} A\left(u_{\epsilon}(t)\right), \varphi(t) \nabla w_{k}\right) \mathrm{d} t\right| \\
& \leq \sup _{t \in(0, T)}|\varphi(t)| \int_{0}^{T}\left\|A\left(u_{\epsilon}(t)\right)\right\|_{\mathbb{L}^{4}(\Omega)}^{3}\left\|\nabla w_{k}\right\|_{\mathbb{L}^{4}(\Omega)} \mathrm{d} t \\
& \leq c\left\|u_{\epsilon}\right\|_{L^{4}\left(0, T ; \mathbb{W}_{0}^{1,4}(\Omega)\right)}^{3}\left\|w_{k}\right\|_{\mathbb{W}_{0}^{1,4}(\Omega)} .
\end{aligned}
$$

From (115)-(117), we find that the nonlinear terms in (113) are continuous with respect to $w_{k} \in \mathbb{W}_{0}^{1,4}(\Omega)$. By a continuity argument, from (113) and (114), we have that, for every $w \in$ $\mathbb{W}_{0}^{1,4}(\Omega)$ and $v \in L^{2}(\Omega)$,

$$
\begin{aligned}
& -\int_{0}^{T}\left\langle u_{\epsilon}(t), w\right\rangle \varphi^{\prime}(t) \mathrm{d} t \\
& \quad+v \int_{0}^{T}\left(\nabla u_{\epsilon}(t), \varphi(t) \nabla w\right) \mathrm{d} t \\
& \quad+\int_{0}^{T} \bar{b}\left(u_{\epsilon}(t), u_{\epsilon}(t), w \varphi(t)\right) \mathrm{d} t \\
& \quad+\alpha \int_{0}^{T}\left(A^{2}\left(u_{\epsilon}(t)\right), \varphi(t) \nabla w\right) \mathrm{d} t \\
& \quad+\beta \int_{0}^{T}\left(\left|A\left(u_{\epsilon}(t)\right)\right|^{2} A\left(u_{\epsilon}(t)\right), \varphi(t) \nabla w\right) \mathrm{d} t \\
& \quad+\int_{0}^{T}\left(\nabla p_{\epsilon}(t), w \varphi(t)\right) \mathrm{d} t=0, \\
& -\epsilon \int_{0}^{T}\left(p_{\epsilon}(t), v\right) \varphi^{\prime}(t) \mathrm{d} t+\int_{0}^{T}\left(\operatorname{div} u_{\epsilon}(t), v \varphi(t)\right) \mathrm{d} t=0 ;
\end{aligned}
$$

that is, in the sense of distribution, we obtain

$$
\begin{aligned}
& \frac{\mathrm{d}}{\mathrm{d} t}\left\langle u_{\epsilon}, w\right\rangle+v\left(\nabla u_{\epsilon}, \nabla w\right)+\bar{b}\left(u_{\epsilon}, u_{\epsilon}, w\right) \\
& \quad+\alpha\left(A^{2}\left(u_{\epsilon}\right), \nabla w\right) \\
& \quad+\beta\left(\left|A\left(u_{\epsilon}\right)\right|^{2} A\left(u_{\epsilon}\right), \nabla w\right)+\left(\nabla p_{\epsilon}, w\right) \\
& =0, \quad \forall w \in \mathbb{W}_{0}^{1,4}(\Omega), \\
& \epsilon \frac{\mathrm{d}}{\mathrm{d} t}\left(p_{\epsilon}, v\right)+\left(\operatorname{div} u_{\epsilon}, v\right)=0, \quad \forall v \in L^{2}(\Omega) .
\end{aligned}
$$

We next prove that the functions $u_{\epsilon}$ and $p_{\epsilon}$ satisfy the initial conditions $u_{\epsilon}(0)=u_{0}$ and $p_{\epsilon}(0)=p_{0}$, respectively. Since $u_{\epsilon} \epsilon$ $L^{2}\left(0, T ; \mathbb{1}_{0}^{1}(\Omega)\right) \cap L^{4}\left(0, T ; \mathbb{W}_{0}^{1,4}(\Omega)\right) \cap L^{\infty}\left(0, T ; \mathbb{L}^{2}(\Omega)\right)$, by $(47)-$ (49), we find that

$$
\begin{gathered}
\bar{B}\left(u_{\epsilon}\right) \in L^{4 / 3}\left(0, T ; \mathbb{\boxplus}^{-1}(\Omega)\right), \\
J\left(u_{\epsilon}\right) \in L^{2}\left(0, T ; \mathbb{W}^{-1}(\Omega)\right), \\
K\left(u_{\epsilon}\right) \in L^{4 / 3}\left(0, T ; \mathbb{\boxplus}^{-1,4 / 3}(\Omega)\right) .
\end{gathered}
$$


Equations (119)-(120) imply that

$$
\begin{aligned}
& \frac{\mathrm{d} u_{\epsilon}}{\mathrm{d} t} \in L^{4 / 3}\left(0, T ; \mathbb{H}^{-1,4 / 3}(\Omega)\right), \\
& \frac{\mathrm{d} p_{\epsilon}}{\mathrm{d} t} \in L^{2}\left(0, T ; L^{2}(\Omega)\right) .
\end{aligned}
$$

Thus by the classical embedding theorem (see $[13,14])$ we have $u_{\epsilon} \in \mathscr{C}\left([0, T] ; \mathbb{L}^{2}(\Omega)\right)$ and $p_{\epsilon} \in \mathscr{C}\left([0, T] ; L^{2}(\Omega)\right)$. To show $u_{\epsilon}(0)=u_{0}$, we take a test function $\varphi(t) \in \mathscr{C}^{1}[0, T]$ with $\varphi(0) \neq 0$ and $\varphi(T)=0$. Then repeating the above limiting process, we obtain

$$
\begin{aligned}
& -\int_{0}^{T}\left\langle u_{\epsilon}, w\right\rangle \varphi^{\prime}(t) \mathrm{d} t+v \int_{0}^{T}\left(\nabla u_{\epsilon}, \varphi(t) \nabla w\right) \mathrm{d} t \\
& \quad+\int_{0}^{T} \bar{b}\left(u_{\epsilon}, u_{\epsilon}, w \varphi(t)\right) \mathrm{d} t+\alpha \int_{0}^{T}\left(A^{2}\left(u_{\epsilon}\right), \nabla w \varphi(t)\right) \mathrm{d} t \\
& \quad+\beta \int_{0}^{T}\left(\left|A\left(u_{\epsilon}\right)\right|^{2} A\left(u_{\epsilon}\right), \nabla w \varphi(t)\right) \mathrm{d} t \\
& \quad+\int_{0}^{T}\left(\nabla p_{\epsilon}, w \varphi(t)\right) \mathrm{d} t \\
& =\left(u_{0}, w\right) \varphi(0), \quad \forall w \in \mathbb{W}_{0}^{1,4}(\Omega), \\
& -\epsilon \int_{0}^{T}\left(p_{\epsilon}, v\right) \varphi^{\prime}(t) \mathrm{d} t+\int_{0}^{T}\left(u_{\epsilon}, \varphi(t) \nabla v\right) \mathrm{d} t \\
& =\left(p_{0}, v\right) \varphi(0), \quad \forall v \in L^{2}(\Omega) .
\end{aligned}
$$

On the other hand, multiplying (119) by $\varphi(t)$ and then integrating, we obtain

$$
\begin{aligned}
& -\int_{0}^{T}\left(u_{\epsilon}, w\right) \varphi^{\prime}(t) \mathrm{d} t+v \int_{0}^{T}\left(\nabla u_{\epsilon}, \varphi(t) \nabla w\right) \mathrm{d} t \\
& \quad+\int_{0}^{T} \bar{b}\left(u_{\epsilon}, u_{\epsilon}, w \varphi(t)\right) \mathrm{d} t \\
& \quad+\alpha \int_{0}^{T}\left(A^{2}\left(u_{\epsilon}\right), \nabla w \varphi(t)\right) \mathrm{d} t \\
& \quad+\beta \int_{0}^{T}\left(\left|A\left(u_{\epsilon}\right)\right|^{2} A\left(u_{\epsilon}\right), \nabla w \varphi(t)\right) \mathrm{d} t \\
& \quad+\int_{0}^{T}\left(\nabla p_{\epsilon}, w \varphi(t)\right) \mathrm{d} t \\
& =\left(u_{\epsilon}(0), w\right) \varphi(0), \quad \forall w \in \mathbb{W}_{0}^{1,4}(\Omega), \\
& -\epsilon \int_{0}^{T}\left(p_{\epsilon}, v\right) \varphi^{\prime}(t) \mathrm{d} t+\int_{0}^{T}\left(\operatorname{div} u_{\epsilon}, v \varphi(t)\right) \mathrm{d} t \\
& =\left(p_{\epsilon}(0), v\right) \varphi(0), \quad \forall v \in L^{2}(\Omega) .
\end{aligned}
$$

We compare (122) with (124) and (123) with (125) to obtain

$$
\begin{gathered}
\left(u_{0}-u_{\epsilon}(0), w\right)=0, \quad \forall w \in \mathbb{W}_{0}^{1,4}(\Omega), \\
\left(p_{0}-p_{\epsilon}(0), v\right)=0, \quad \forall v \in L^{2}(\Omega) .
\end{gathered}
$$

Hence $u_{\epsilon}(0)=u_{0}$ and $p_{\epsilon}(0)=p_{0}$. The uniqueness of the solutions is proved exactly the same as Lemma 3.4 of [7]. The proof of Theorem 9 now is complete.

\section{Convergence of Solutions for the Slightly Compressible Third Grade Fluids to the Solutions of Incompressible Third Grade Fluids}

In this section, we shall show that the solutions of the slightly compressible third grade fluids equations converge to the solutions of the incompressible third grade fluids equations. Let $\left\{u_{\epsilon}, p_{\epsilon}\right\}_{\epsilon \in(0,1]}$ be the solutions of the slightly compressible third grade fluids equations. We prove that there exists a sequence $\left\{\epsilon_{n}\right\}_{n=1}^{\infty} \subset(0,1]$ converging to $0^{+}$such that the sequence $\left\{u_{\epsilon_{n}}, p_{\epsilon_{n}}\right\}$ is convergent. Let $\left\{u_{*}, p_{*}\right\}$ be its limit. We prove that $\left\{u_{*}, p_{*}\right\}$ is a solution of the incompressible third grade fluids equations.

Theorem 10. For any given $u_{0} \in H$ and $p_{0} \in L^{2}(\Omega)$, there exists a sequence $\left\{\epsilon_{n}\right\}_{n=1}^{\infty} \subset(0,1]$ such that $\epsilon_{n} \rightarrow 0^{+}$as $n \rightarrow$ $\infty$ and the solutions $\left\{u_{\epsilon_{n}}, p_{\epsilon_{n}}\right\}$ of Problem 7 are convergent. Let $\left\{u_{*}, p_{*}\right\}$ be its limit. Then $\left\{u_{*}, p_{*}\right\}$ is a solution of the incompressible third grade fluids equations (5). Moreover,

$$
\begin{gathered}
u_{\epsilon} \stackrel{\text { weak-star }}{\longrightarrow} u_{*} \quad \text { in } L^{\infty}\left(0, T ; \mathbb{L}^{2}(\Omega)\right), \\
u_{\epsilon} \stackrel{\text { weakly }}{\longrightarrow} u_{*} \quad \text { in } L^{2}\left(0, T ; \mathbb{T}_{0}^{1}(\Omega)\right), \\
u_{\epsilon} \stackrel{\text { strongly }}{\longrightarrow} u_{*} \quad \text { in } L^{2}\left(0, T ; \mathbb{L}^{2}(\Omega)\right), \\
\nabla p_{\epsilon} \stackrel{\text { weakly }}{\longrightarrow} \nabla p_{*} \text { in } L^{4 / 3}\left(0, T ; \mathbb{W}^{-1,4 / 3}(\Omega)\right) .
\end{gathered}
$$

Proof. By (78)-(80) and (98), we have for all $\epsilon \in(0,1]$ the following:

$$
\begin{gathered}
\left\|u_{\epsilon}\right\|_{L^{\infty}\left(0, T ; \mathbb{L}^{2}(\Omega)\right)} \leq \liminf _{n \rightarrow \infty}\left\|u_{\epsilon n}\right\|_{L^{\infty}\left(0, T ; \mathbb{L}^{2}(\Omega)\right)} \leq \sqrt{d}, \\
\left\|u_{\epsilon}\right\|_{L^{2}\left(0, T ; \mathbb{N}_{0}^{1}(\Omega)\right)} \leq \liminf _{n \rightarrow \infty}\left\|u_{\epsilon n}\right\|_{L^{2}\left(0, T ; \mathbb{1}_{0}^{1}(\Omega)\right)} \leq \sqrt{\frac{d}{2 \nu \sigma_{0}}}, \\
\sqrt{\epsilon}\left\|p_{\epsilon}\right\|_{L^{\infty}\left(0, T ; L^{2}(\Omega)\right)} \leq \liminf _{n \rightarrow \infty} \sqrt{\epsilon}\left\|p_{\epsilon n}\right\|_{L^{\infty}\left(0, T ; L^{2}(\Omega)\right)} \leq \sqrt{d},
\end{gathered}
$$

$$
\int_{-\infty}^{+\infty}|\tau|^{2 \gamma}\left\|\widehat{u}_{\epsilon}(\tau)\right\|^{2} \mathrm{~d} \tau \leq \liminf _{n \rightarrow \infty} \int_{-\infty}^{+\infty}|\tau|^{2 \gamma}\left\|\widehat{u}_{\epsilon n}(\tau)\right\|^{2} \mathrm{~d} \tau \leq c .
$$


In terms of (131)-(134), there exist a sequence $\left\{\epsilon_{n}\right\} \subset(0,1]$ $\left(\epsilon_{n} \rightarrow 0^{+}\right.$as $\left.n \rightarrow \infty\right)$ and some $u_{*} \in L^{\infty}\left(0, T ; \mathbb{L}^{2}(\Omega)\right) \cap$ $L^{2}\left(0, T ; \mathbb{Q}_{0}^{1}(\Omega)\right)$ and $p_{*} \in L^{\infty}\left(0, T ; L^{2}(\Omega)\right)$ such that when $\epsilon_{n} \rightarrow 0^{+}$

$$
\begin{aligned}
& u_{\epsilon_{n}} \stackrel{\text { weak-star }}{\longrightarrow} u_{*} \text { in } L^{\infty}\left(0, T ; \mathbb{L}^{2}(\Omega)\right) \text {, } \\
& u_{\epsilon_{n}} \stackrel{\text { weakly }}{\longrightarrow} u_{*} \quad \text { in } L^{2}\left(0, T ; \mathbb{Q}_{0}^{1}(\Omega)\right) \text {, } \\
& u_{\epsilon_{n}} \stackrel{\text { strongly }}{\longrightarrow} u_{*} \text { in } L^{2}\left(0, T ; \mathbb{L}^{2}(\Omega)\right) \text {, } \\
& \sqrt{\epsilon_{n}} p_{\epsilon_{n}} \stackrel{\text { weak-star }}{\longrightarrow} p_{*} \quad \text { in } L^{\infty}\left(0, T ; L^{2}(\Omega)\right) \text {. }
\end{aligned}
$$

Passing to the limit in (57) in the sense of distribution as $\epsilon_{n} \rightarrow$ $0^{+}$, we obtain

$$
\epsilon_{n} \frac{\mathrm{d}}{\mathrm{d} t}\left(p_{\epsilon_{n}}, q\right)=\sqrt{\epsilon_{n}} \sqrt{\epsilon_{n}} \frac{\mathrm{d}}{\mathrm{d} t}\left(p_{\epsilon_{n}}, q\right) \longrightarrow 0, \quad \forall q \in L^{2}(\Omega) .
$$

Equations (57) and (139) give

$$
\left(\operatorname{div} u_{*}, q\right)=0, \quad \forall q \in L^{2}(\Omega),
$$

which implies that $\operatorname{div} u_{*}=0$ and thus

$$
u_{*} \in L^{2}(0, T ; V) \cap L^{\infty}(0, T ; H) .
$$

Now let $w \in \mathbb{W}_{0}^{1,4}(\Omega)$. Since $\left(\nabla p_{\epsilon}, w\right)=-\left(p_{\epsilon}, \operatorname{div} w\right)=0$, (56) shows

$$
\begin{gathered}
\frac{\mathrm{d}}{\mathrm{d} t}\left\langle u_{\epsilon}, w\right\rangle-v\left\langle\mathscr{L} u_{\epsilon}, w\right\rangle+\bar{b}\left(u_{\epsilon}, u_{\epsilon}, w\right) \\
+J\left(u_{\epsilon}, w\right)+\beta\left(K\left(u_{\epsilon}\right), w\right)=0 .
\end{gathered}
$$

Let $\varphi(t) \in \mathscr{C}_{0}^{\infty}(0, T)$. Multiplying (142) by $\varphi(t)$, integrating in $t$ the resulting equality over $(0, T)$, and then using integration by parts, we obtain

$$
\begin{aligned}
& -\int_{0}^{T}\left\langle u_{\epsilon}(t), w\right\rangle \varphi^{\prime}(t) \mathrm{d} t \\
& \quad+v \int_{0}^{T}\left(\nabla u_{\epsilon}(t), \varphi(t) \nabla w\right) \mathrm{d} t \\
& \quad+\int_{0}^{T} \bar{b}\left(u_{\epsilon}(t), u_{\epsilon}(t), w \varphi(t)\right) \mathrm{d} t \\
& \quad+\alpha \int_{0}^{T}\left(A^{2}\left(u_{\epsilon}(t)\right), \varphi(t) \nabla w\right) \mathrm{d} t \\
& =-\beta \int_{0}^{T}\left(\left|A\left(u_{\epsilon}(t)\right)\right|^{2} A\left(u_{\epsilon}(t)\right), \varphi(t) \nabla w\right) \mathrm{d} t
\end{aligned}
$$

$\forall w \in \mathbb{W}_{0}^{1,4}(\Omega)$
Using the convergent relations (135)-(138) and the similar derivations of (111)-(112), we can obtain

$$
\begin{gathered}
\int_{0}^{T}\left(J\left(u_{\epsilon_{n}}(t)\right), w_{k} \varphi(t)\right) \mathrm{d} t \\
\longrightarrow \int_{0}^{T}\left(J\left(u_{*}(t)\right), w_{k} \varphi(t)\right) \mathrm{d} t, \\
\int_{0}^{T}\left(K\left(u_{\epsilon_{n}}(t)\right), w_{k} \varphi(t)\right) \mathrm{d} t \\
\longrightarrow \int_{0}^{T}\left(K\left(u_{*}(t)\right), w_{k} \varphi(t)\right) \mathrm{d} t, \\
\int_{0}^{T} \bar{b}\left(u_{\epsilon_{n}}(t), u_{\epsilon_{n}}(t), w_{k} \varphi(t)\right) \mathrm{d} t \\
\longrightarrow \int_{0}^{T} \bar{b}\left(u_{*}(t), u_{*}(t), w_{k} \varphi(t)\right) \mathrm{d} t \\
=\int_{0}^{T} b\left(u_{*}(t), u_{*}(t), w_{k} \varphi(t)\right) \mathrm{d} t,
\end{gathered}
$$

since $\operatorname{div} u_{*}=0$ and $\bar{b}\left(u_{*}, u_{*}, w_{k} \varphi(t)\right)=b\left(u_{*}, u_{*}, w_{k} \varphi(t)\right)$. Also by the convergent relations (135)-(138) and (144)-(146), we can pass to the limit for $\epsilon_{n}$ in (143) and obtain

$$
\begin{aligned}
-\int_{0}^{T} & \left\langle u_{*}(t), w\right\rangle \varphi^{\prime}(t) \mathrm{d} t+v \int_{0}^{T}\left(\nabla u_{*}(t), \varphi(t) \nabla w\right) \mathrm{d} t \\
& +\int_{0}^{T} b\left(u_{*}(t), u_{*}(t), w \varphi(t)\right) \mathrm{d} t \\
& +\int_{0}^{T}\left(J\left(u_{*}(t)\right), w \varphi(t)\right) \mathrm{d} t \\
= & -\beta \int_{0}^{T}\left(K\left(u_{*}(t)\right), w \varphi(t)\right) \mathrm{d} t, \quad \forall w \in \mathbb{W}_{0}^{1,4}(\Omega) .
\end{aligned}
$$

The relation (147) is the same as (27). Then it is easy to show that $u_{*}$ is a solution of Problem 1. Correspondingly, $\left\{u_{*}, p_{*}\right\}$ satisfies (5).

We next prove the convergent relations (127)-(130). Equation (127) follows easily from (131) and (128) follows easily from (132). Equation (129) can be deduced from (134)(136) and the compact embedding theorem (see Lemma 8). It remains to prove (130). To this end, we write (56) as

$$
\nabla p_{\epsilon_{n}}=-u_{\epsilon_{n}}^{\prime}-\nu \mathscr{L} u_{\epsilon_{n}}-\bar{B}\left(u_{\epsilon_{n}}\right)-J\left(u_{\epsilon_{n}}\right)-\beta K\left(u_{\epsilon_{n}}\right) \text {. }
$$

The convergent results for $u_{\epsilon_{n}}$ show that the right-hand side of (148) converges weakly to

$$
-u_{*}^{\prime}-v A u_{*}-B\left(u_{*}\right)-J\left(u_{*}\right)-\beta K\left(u_{*}\right)
$$

in $L^{4 / 3}\left(0, T ; \mathbb{W}^{-1,4 / 3}(\Omega)\right)$ as $\epsilon_{n} \rightarrow 0^{+}$. We find that (149) is exactly $\nabla p_{*}$. Therefore,

$$
\nabla p_{\epsilon_{n}} \stackrel{\text { weakly }}{\longrightarrow} \nabla p_{*} \quad \text { in } L^{4 / 3}\left(0, T ; \mathbb{W}^{-1,4 / 3}(\Omega)\right) .
$$

The proof is complete. 


\section{Concluding Remarks}

In this study, we discuss the approximation of a class of incompressible third grade fluids equations by the artificial compressibility method. We first introduce a family of perturbed slightly compressible third grade fluids equations (depending on a positive parameter $\epsilon$ ) which approximate the incompressible equations as $\epsilon \rightarrow 0^{+}$. Then we prove the existence and uniqueness of weak solutions for the slightly compressible equations. The obtained weak solution provides us with the "approximation" of the original incompressible equations. The merit of the solution is that it overcomes the computational difficulties connected with the constraint "div $u=0$." It is easier to be implemented in the simulation than in the original incompressible equations as the constraint " $\operatorname{div} u=0$ " has been replaced by the evolution equation $\epsilon \partial_{t} p_{\epsilon}+\operatorname{div} u_{\epsilon}=0$ (see, e.g., [13]). Lastly, we establish the fact that the solutions of the slightly compressible equations converge to the solutions of the incompressible equations as $\epsilon \rightarrow 0^{+}$. This convergence indicates that the approximation is reasonable.

There is still much work to be done concerning the third grade fluids equations. For example, we could consider borrowing the approaches of [17-20], the existence and regularity of the uniform attractors and pullback attractors, and also the existence and limiting behavior of the trajectory attractor for the the third grade fluids equations when its solutions could not be unique. These problems will be the topics of some other papers.

\section{Conflict of Interests}

The authors declare that there is no conflict of interests regarding the publication of this paper.

\section{Acknowledgments}

The authors thank warmly the anonymous referee for his/her pertinent comments and suggestions, which greatly improve the earlier paper. This work was supported by the National Program on Key Basic Research Project of China (no. 2015CB057905) and the National Natural Science Foundation of China (nos. 51279202, 91215301, and U1402231).

\section{References}

[1] R. S. Rivlin and J. L. Ericksen, "Stress-deformation relations for isotropic materials," Journal of Rational Mechanics and Analysis, vol. 4, pp. 323-425, 1955.

[2] T. Aziz and A. Aziz, "MHD flow of a third grade fluid in a porous half space with plate suction or injection: an analytical approach," Applied Mathematics and Computation, vol. 218, no. 21, pp. 10443-10453, 2012.

[3] T. Aziz, F. M. Mahomed, and A. Aziz, "Group invariant solutions for the unsteady MHD flow of a third grade fluid in a porous medium," International Journal of Non-Linear Mechanics, vol. 47, no. 7, pp. 792-798, 2012.

[4] T. Aziz, F. M. Mahomed, M. Ayub, and D. P. Mason, "Non-linear time-dependent flow models of third grade fluids: a conditional symmetry approach," International Journal of Non-Linear Mechanics, vol. 54, pp. 55-65, 2013.

[5] V. Busuioc and D. Iftimie, "Global existence and uniqueness of solutions for the equations of third grade fluids," International Journal of Non-Linear Mechanics, vol. 39, no. 1, pp. 1-12, 2004.

[6] D. Cioranescu and V. Girault, "Weak and classical solutions of a family of second grade fluids," International Journal of NonLinear Mechanics, vol. 32, no. 2, pp. 317-335, 1997.

[7] M. Hamza and M. Paicu, "Global existence and uniqueness result of a class of third-grade fluids equations," Nonlinearity, vol. 20, no. 5, pp. 1095-1114, 2007.

[8] M. Paicu, G. Raugel, and A. Rekalo, "Regularity of the global attractor and finite-dimensional behavior for the second grade fluid equations," Journal of Differential Equations, vol. 252, no. 6, pp. 3695-3751, 2012.

[9] A. Sequeira and J. Videman, "Global existence of classical solutions for the equations of third grade fluids," Journal of Mathematical and Physical Sciences, vol. 29, no. 2, pp. 47-69, 1995.

[10] C. Zhao, Y. Liang, and M. Zhao, "Upper and lower bounds of time decay rate of solutions to a class of incompressible third grade fluid equations," Nonlinear Analysis: Real World Applications, vol. 15, pp. 229-238, 2014.

[11] R. A. Adams, Sobolev Spaces, Academic Press, New York, NY, USA, 1975.

[12] R. Temam, Infinite Dimensional Dynamical Systems in Mechanics and Physics, Springer, Berlin, Germany, 2nd edition, 1997.

[13] R. Temam, Navier-Stokes Equations (Theory and Numerical Analysis), North-Holland, Amsterdam, Netherlands, 1984.

[14] V. V. Chepyzhov and M. I. Vishik, Attractors for Equations of Mathematical Physics, vol. 49 of AMS Colloquium Publications, American Mathematical Society, Providence, RI, USA, 2002.

[15] C. Zhao and Y. You, "Approximation of the incompressible convective Brinkman-Forchheimer equations," Journal of Evolution Equations, vol. 12, no. 4, pp. 767-788, 2012.

[16] C. Zhao, "Approximation of the incompressible non-newtonian fluid equations by the artificial compressibility method," Mathematical Methods in the Applied Sciences, vol. 36, no. 7, pp. 840856, 2013.

[17] C. Zhao, "Pullback asymptotic behavior of solutions for a non-autonomous non-Newtonian fluid on two-dimensional unbounded domains," Journal of Mathematical Physics, vol. 53, no. 12, Article ID 122702, pp. 1-21, 2012.

[18] C. Zhao, "Dynamics of non-autonomous equations of nonnewtonian fluid on 2D unbounded domains," Dynamics of Partial Differential Equations, vol. 10, no. 3, pp. 283-312, 2013.

[19] C. Zhao, G. Liu, and W. Wang, "Smooth pullback attractors for a non-autonomous 2D non-Newtonian fluid and their tempered behaviors," Journal of Mathematical Fluid Mechanics, vol. 16, no. 2, pp. 243-262, 2014.

[20] C. Zhao, L. Kong, G. Liu, and M. Zhao, "The trajectory attractor and its limiting behavior for the convective BrinkmanForchheimer equations," Topological Methods in Nonlinear Analysis. In press. 


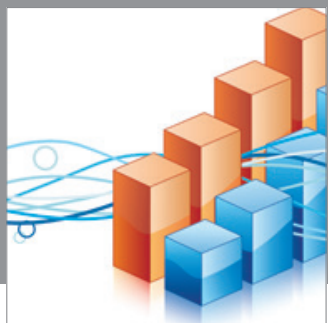

Advances in

Operations Research

mansans

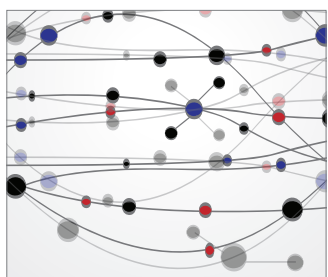

The Scientific World Journal
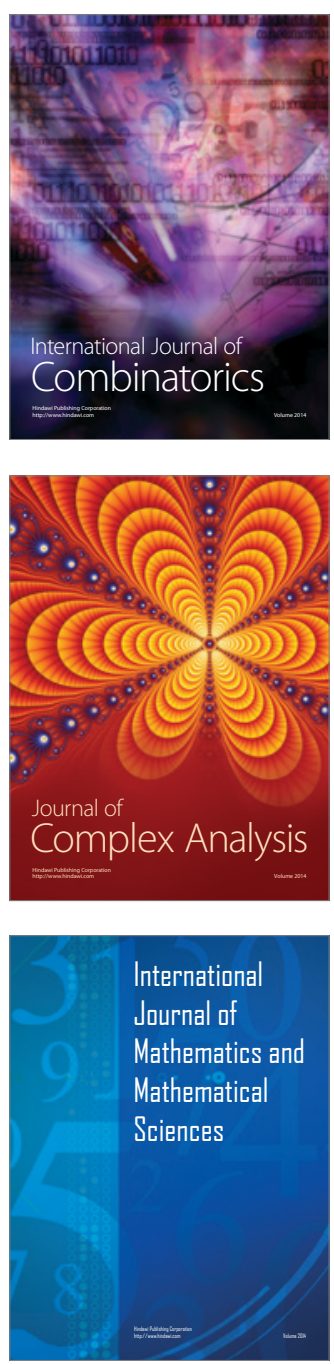
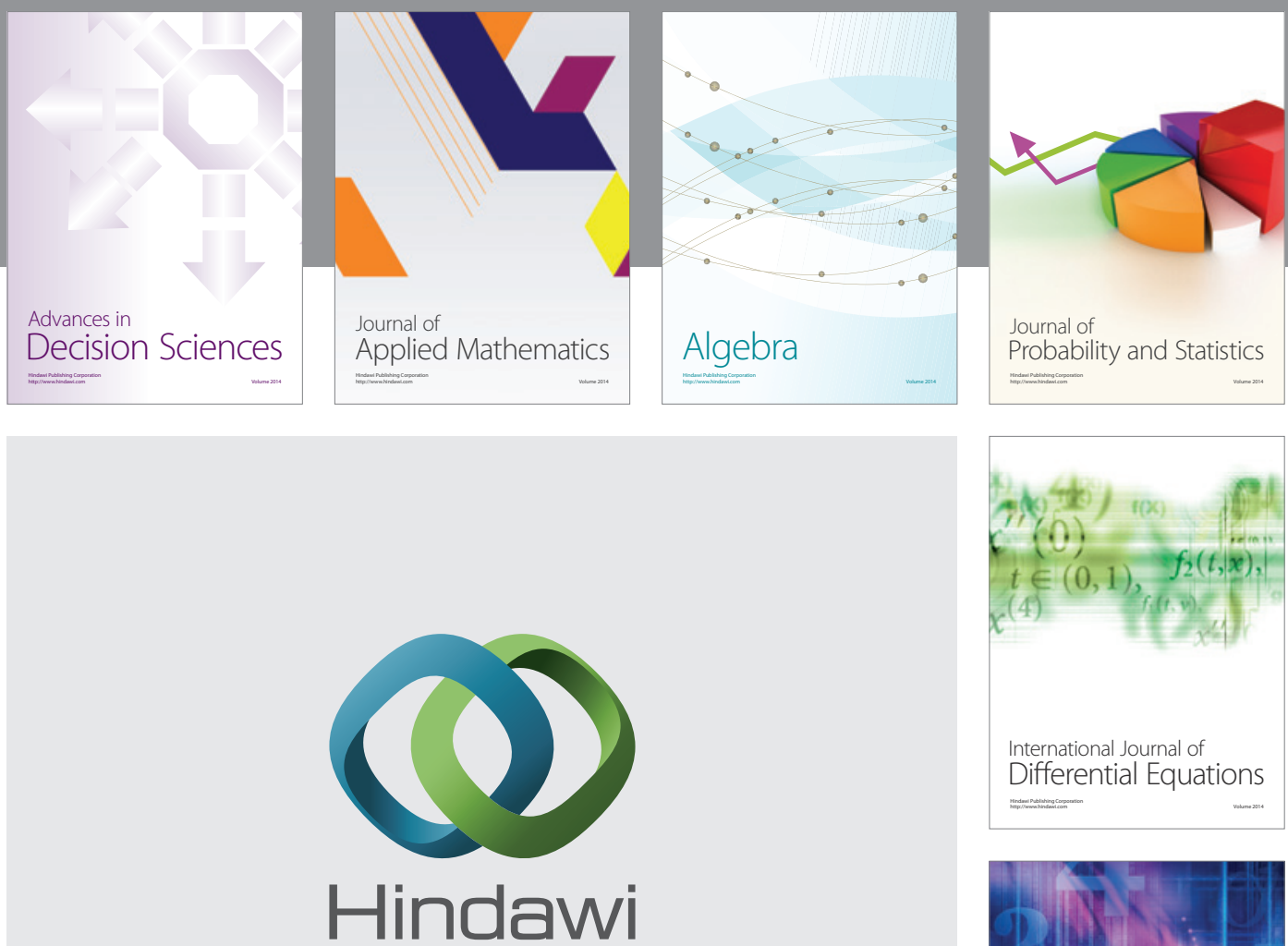

Submit your manuscripts at http://www.hindawi.com
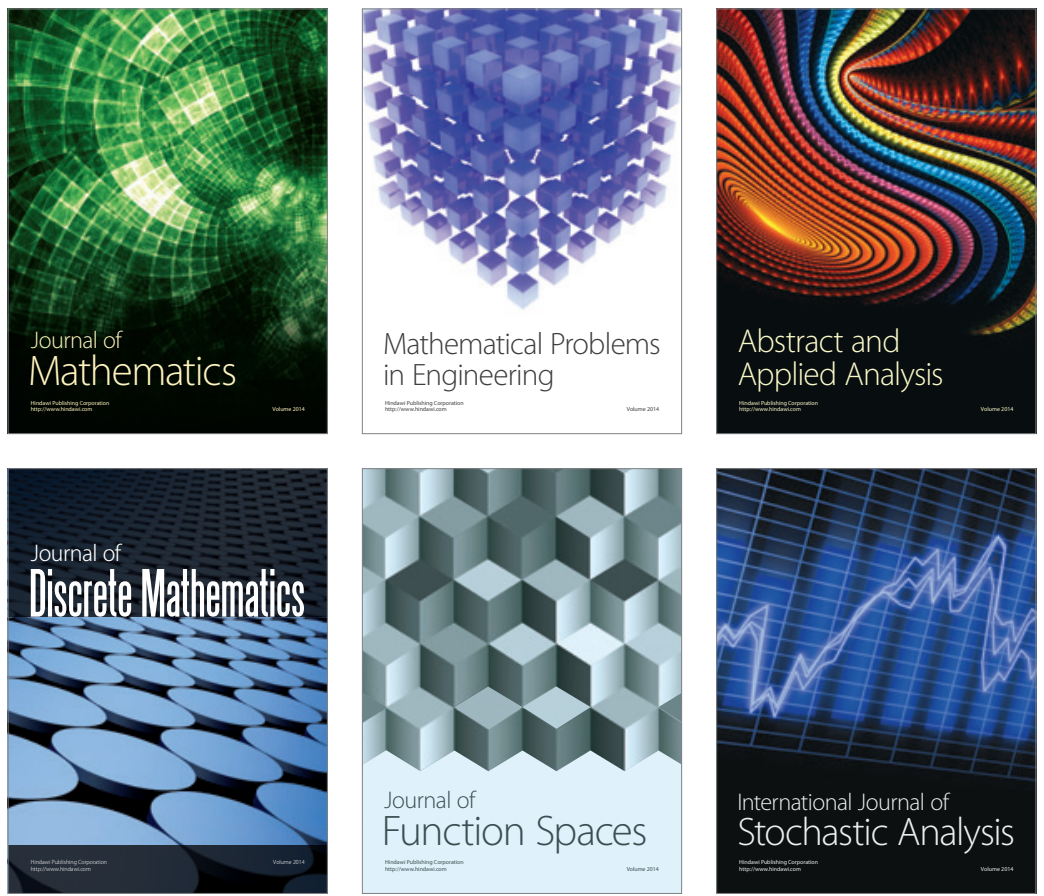

Journal of

Function Spaces

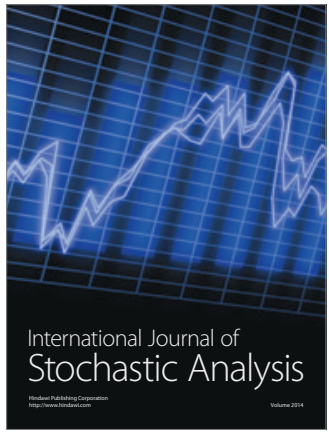

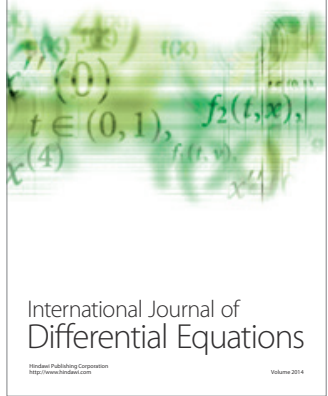
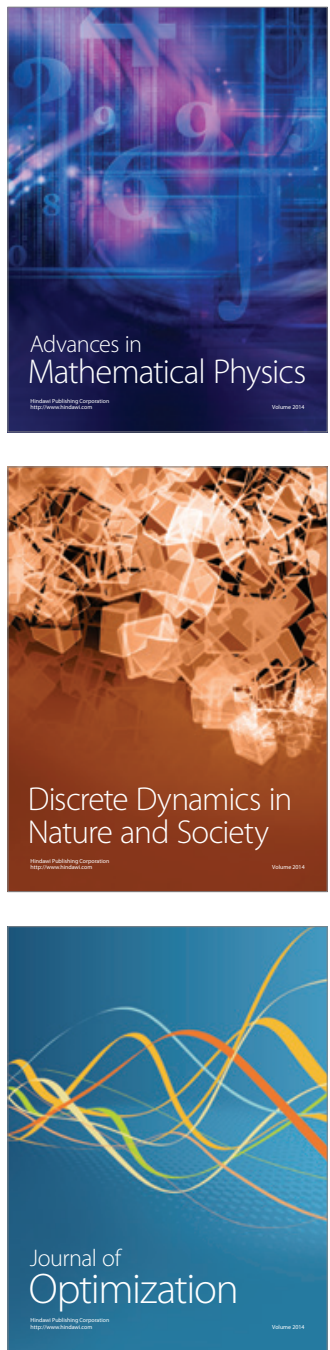